\title{
Stability analysis and decentralized control of inverter-based ac microgrid
}

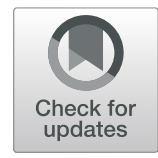

\author{
Mehdi Farokhian Firuzi (1) ${ }^{*}$, Alireza Roosta and Mohsen Gitizadeh
}

\begin{abstract}
This work considers the problem of decentralized control of inverter-based ac micro-grid in different operation modes. The main objectives are to (i) design decentralized frequency and voltage controllers, to gather with power sharing, without information exchange between microsources (ii) design passive dynamic controllers which ensure stability of the entire microgrid system (iii) capture nonlinear, interconnected and large-scale dynamic of the micro-grid system with meshed topology as a port-Hamiltonian formulation (iv) expand the property of shifted-energy function in the context of decentralized control of ac micro-grid ( $v$ ) analysis of system stability in large signal point of view. More precisely, to deal with nonlinear, interconnected and large-scale structure of micro-grid systems, the port-Hamiltonian formulation is used to capture the dynamic of micro-grid components including microsource, distribution line and load dynamics as well as interconnection controllers. Furthermore, to deal with large signal stability problem of the microgrid system in the grid-connected and islanded conditions, the shifted-Hamiltonian energy function is served as a storage function to ensure incremental passivity and stability of the microgrid system. Moreover, it is shown that the aggregating of the microgrid dynamic and the decentralized controller dynamics satisfies the incremental passivity. Finally, the effectiveness of the proposed controllers is evaluated through simulation studies. The different scenarios including grid-connected and islanded modes as well as transition between both modes are simulated. The simulation conforms that the decentralized control dynamics are suited to achieve the desired objective of frequency synchronization, voltage control and power sharing in the grid-connected and islanded modes. The simulation results demonstrate the effectiveness of the proposed control strategy.
\end{abstract}

Keywords: Decentralized control, Inverter-based ac micro-grid, Frequency and voltage control, Active and reactive power sharing, Incremental passivity, Port-Hamiltonian framework, Shifted-energy function

\section{Introduction}

In recent years, renewable energy systems have been increasingly employed to mainly reduce the cost of energy prices and solve the environmental issues. A cluster of elements such as loads, distributed generators (DG), distributed storage units and controllable loads, connected through a medium or low voltage interconnecting power grid is defined as a Microgrid system [1,2]. When the number of elements in each microgrid is increased, the problem of controlling them can be very challenging in a centralized way. Using communication links, especially in large-scale system, might not be economical and practical. Moreover, due to the communication link delay or failure, the reliability of the system may deteriorate. Thus,

*Correspondence: m.farokhian@sutech.ac.ir Shiraz University of Technology, Shiraz, Iran each microgrid has the responsibility of controlling their own units locally. From the decentralized point of view, the controller of microsource units only needs the local variables [3].

Different decentralized control strategies including droop-based and non-droop-based controllers have been proposed for $a c$ microgrid systems with different operation modes. Early work on decentralized parallel inverter control concepts suitable for microgrid operation was reported in [4]. Subsequent work [5, 6] extended the droop concept to ensure sharing of harmonic currents of non-linear loads. In further investigation of the droop concept, some researchers [7-9] have proposed powerangle droop control, in which the phase angle of the distributed source voltage, relative to a system-wide common timing reference, is set according to a droop law. A static 
droop compensator is utilized for power sharing in [10]. An enhanced droop control featuring a transient droop performance is proposed [11]. To improve the active and reactive power decoupling performance, improved droop controllers with virtual output impedance are reported $[11,12]$. To account for nonlinear loads, harmonic-based droop controllers are investigated [13, 14]. To realize realtime decentralized control strategies a decentralized control strategy extracted from off-line optimization results is designed [15], but this control design requires prior knowledge of the grid structure and extensive computations are needed if the grid structure changes. Furthermore, the methods proposed in $[16,17]$ allow for the seamless plugging-in, unplugging and replacement of microsource units without spoiling microgrid stability. Control design procedures with these features have been termed plug-and-play [16, 18-21]. However, existing droop controllers are synthesized in the sense of the small-signal model of the power transfer mechanisms. To improve the performance of parallel operation, a stationary-reference-frame droop-controlled model of parallel-connected inverters is introduced and inner voltage and current control loops are proposed [22]. Decoupled droop control techniques are proposed and analyzed in [23] to obtain independent association of frequency with active power and voltage with reactive power. To exploit the flexibility and fast dynamics of the inverterbased distributed energy resources, a piecewise linear voltage-current droop controller is introduced [24]. To improve the reactive power sharing performance of droop control, a virtual impedance optimization method for reactive power sharing in networked microgrid is proposed [25]. To control parallel-connected Inverters, a synchronous reference frame virtual impedance loop is proposed [26]. To soft synchronization of microgrid, an approach using robust control theory is reported in [27]. In [28], a reactive-power sharing scheme for two inverter-based distributed generations with unequal line impedances in islanded microgrids is reported. An adaptative droop control for balancing the state of charge of multiple energy storage systems in decentralized microgrids is studied in [29]. Moreover, numerous control methodologies have been proposed to improve the $a c$ microgrid performance [30-37]. A fully decentralized control and a $f \quad P / Q$ droop control scheme of gridconnected cascaded inverters are reported [30, 31]. A cost-function-based decentralized power quality compensation method realizing simultaneously bus voltage compensation and inverter current sharing is proposed [32]. A dual control strategy for power sharing improvement in islanded mode of AC microgrid is reported [33]. A decentralized optimal servo control system for implementing instantaneous reactive power sharing in microgrids is proposed [34]. In [38], a passivity-based decentralized control strategy for current-controlled inverters in $a c$ microgrids is reported. an adaptive droop control is proposed including a fuzzy-based droop coefficient adjustment in [35]. Decentralized parallel operation of single phase inverters in islanded mode is reported in [36]. In the study by [37], two-stage adaptive virtual resistor (AVR) control scheme for low-voltage parallel inverters is proposed. A decentralized control with unique equilibrium point for cascaded-type microgrid is proposed in [39]. By far the largest body of research work done in decentralized microgrid control has been for radial architectures (see also [3]). Notably, the droop control has several limitations and challenges including: inability to guarantee large signal stability, weak transient performance, weak performance for mixed-line microgrids with resistive-inductive line conditions, and coupled dynamics between active and reactive power.

Early work towards stability analysis of decentralized controlled intverters based on droop-control was reported in [40]. A generalized approach for analyzing the small-signal stability of interconnected inverter systems was proposed. This result was extended in [41] with the inclusion of reactive power-voltage magnitude droops for the interface inverters. In [42], the authors propose a method, based on the operating point, to set droop gains adaptively. A computational approach to determining radial microgrid stability, scalable to large systems, is presented in [43]. Moreover, numerous methodologies have been proposed to enhance the microgrid stability performance via modifying the inverter control loops [44-48]. These techniques and methods include a combination of repetitive and deadbeat control with feedforward compensation for disturbance rejection [46], secondary control based on potential-function [47], real-time small signal stability analysis for droop gain schedule [45] and droop gain variation for increasing virtual inertia [48]. The various analytical stability tools for microgrid controllers design are reported in [49-56]. The small signal models of the inverter based microgrid is established in [50, 51, 56]. The active load impacts on system small-signal stability are analyzed in [52], it shows the active loads with a large $d c$ voltage controller gain may lead to instability. A combination of small-signal and time-domain simulation for a comprehensive stability analysis are used in $[49,53,57-59]$. Another impedance-based stability criterion for grid-connected inverters is reported in [55]. The frequency regulation of microgrid by utilizing the kinetic energy from the induction motor loads is studied in [60]. An adaptive droop adjustment based on the microgrid small signal model is introduced in [42]. The authors in [61] use the on-line grid parameter estimation combined with small signal stability analysis to detect the islanding and adjust the inverters droop control parameters. The 
droop scheduling scheme based on bifurcation theory is presented in [62] to find the worst primary reserve share that is closest to instability. A mode-adaptive droop control method is proposed in [63]. Sufficient conditions for voltage stability in a droop-controlled lossy microgrid is reported in [64]. Furthermore, sufficient conditions for stability of lossless microgrids based on energy function are established in [65]. Two nonlinear droop controls are proposed in $[66,67]$ to enhance microgrid frequency regulation. Nonetheless, it may either require multiple small signal stability analyzes to determine the acceptable droop gains [66] or lead to instability due to a high droop gain when the frequency is close to the nominal value $[67,68]$. Moreover, the complete reviews and classifications of stability in microgrids are presented [69-71].

In addition to the droop-based control strategies, nondroop-based approaches for voltage and frequency control of the islanded microgrids have also been developed, e.g. [72-77, 77-80]. A robust servomechanism controller for a single-DG/ Multi-DG islanded system, which uses the internal oscillator to control the frequency in an open-loop manner, has been proposed in [73, 74, 78, 79]. In addition, the following methods have been proposed for single-DER microgrids: a voltage controller, designed using an $H_{\infty}$ approach and repetitive control technique, to mitigate voltage harmonics of the point of common coupling (PCC) [81] and a robust control scheme for a microgrid designed based on an $H_{\infty}$ approach to provide a robust performance [82]; and a robust servomechanism approach for PCC voltage control [73]. However, these methods are only applicable to single-DER microgrids. In the study by [83], a decentralized servomechanism controller based on robust approach for the islanded operation of radial connection of two distributed generation (DG) units is proposed. In [84] state feedback is combined with a decentralized LMI strategy to ensure stabilization and frequency regulation, while [85] studies the performance of decentralized frequency-control algorithm based on integral action. To improve current-sharing, a robust controller for parallel-connected inverter-based DGs in lossy microgrid networks is reported in [86]. Although extensive research has been carried out on the development of non-droop-based control of microgrids, they suffer from one or more of the following drawbacks: in applicability to multiple-microsources, inability to guarantee stability and/or performance with respect to several microsources, and high-order controller structures.

To overcome the disadvantages of the existing microgrid control approaches, various challenges associated with large signal stability, seamless transition between different operation modes and low-complexity of the local controllers must be addressed.
In this study, to deal with nonlinear, interconnected and large-scale structures of microgrid systems, the port-Hamiltonian formulation is used to capture the dynamic of microgrid components and interconnection controllers. In addition, the decentralized controllers are also formulated as port-Hamiltonian systems that are connected to the microsources with interaction ports. This framework describes the dynamics in terms of the system stored energy, interconnection and dissipation structures [87-89]. Furthermore, we focus on a more accurate and higher order model for the inverter-based microsources than conventionally used in the literature $[65,80,90]$.

In this work, to deal with large signal stability problem of microgrid system, the shifted-Hamiltonian energy function is served as a storage function to ensure incrementalpassivity of system. Notably, the large signal stability analysis is necessary to guarantee seamless transition between islanded/grid-connected modes and the convergence of equilibrium states of nonlinear microgrid dynamic. Although historically energy functions have played a crucial role to cope with accurate models of power systems (see also [80, 90-97]), our approach based on the incremental passivity allows us to cover an accurate dynamic of microgrids and paves the way for the design of decentralized controllers.

In the reported research works, the microgrids have been almost studied in small-signal point of view while no solution has been proposed regarding the controller to face with large-signal stability. In this paper, authors aim to model the microgrid components and propose new decentralized passivity-based control strategy for an microgrid consisting microsource units and local loads based on port-Hamiltonian framework, shifted-energy function, and incremental passivity to ensure stability of the entire system and enhance its performance for transition between grid-connected to islanded modes and guarantee the desired frequency synchronization, voltage tracking as well as power sharing. This paper presents theoretical concepts, requirements, and necessary conditions for stability and performance of microgrid with meshed topology. Finally, in addition to stability and performance analysis, the effectiveness of the proposed controller is evaluated by set of simulation studies.

More precisely, the main contributions of the paper are six-fold:

- Design decentralized frequency and voltage controllers, to gather with power sharing, without information exchange between microsources and their local controllers,

- Design passive dynamic controllers which ensure stability of the entire microgrid system,

- Capture nonlinear, interconnected and large-scale dynamic of the micro-grid system with meshed topology as a port-Hamiltonian formulation, 
- Expand the property of shifted-energy function in the context of decentralized control of micro-grid,

- Serve the shifted-Hamiltonian energy function as a storage function to ensure incremental passivity,

- Analysis of system stability in large signal point of view.

The remainder of this paper is organized as follows. In Section 2, the port-Hamiltonian formulation and shifted-Hamiltonian energy function as well as incremental passivity are defined as a background of this study. The microgrid topology and structure are presented in Section 3. In Section 4, the microgrid component dynamics are also formulated. In addition, the microgrid units (e.g. microsource, load and distribution line) are modeled based on port Hamiltonian formulation. The open-loop analysis of overall microgrid is established in Section 5. In Sections 6 and 7, the different passive controllers for frequency and voltage control are also proposed and analyzed in different operation modes. Furthermore, to deal with large signal stability problem, the shifted-Hamiltonian energy function is served as a storage function to ensure incremental passivity of entire system. Finally, the paper is closed with simulation results in Section 8 and conclusions remarks as well as discussion of future work in Section 9.

\section{Background: port-Hamiltonian and incremental passivity formulation}

As mentioned, to deal with nonlinear, interconnected and large-scale structures of microgrid systems, the port-Hamiltonian formulation is used to capture the dynamic of microgrid components and interconnection controllers. In addition, the decentralized controllers are also formulated as port-Hamiltonian systems that are connected to the microsources with interaction ports. This framework describes the dynamics in terms of the system stored energy, interconnection and dissipation structures [87-89]. Moreover, the port-Hamiltonain formulation is described as follows:

Definition 1 A port-Hamiltonian system (in inputstate-output form) is given by:

$$
\begin{aligned}
& \dot{x}=(\mathcal{J}(x)-\mathcal{R}(x)) \nabla \mathcal{H}(x)+g(x) u, \\
& y=g^{\top}(x) \nabla \mathcal{H}(x) .
\end{aligned}
$$

With state $x \in \mathbb{R}^{n}$, input $u \in \mathbb{R}^{m}$ and output $y \in \mathbb{R}^{m}$ [87, 88]. In addition, $\mathcal{J}^{\top}(x)=-\mathcal{J}(x)$ is interconnection matrix, $\mathcal{R}^{\top}(x)=\mathcal{R}(x) \geq 0$ is dissipation matrix, and $\nabla \mathcal{H}(x)$ is also the vector of partial derivatives of the Hamiltonian energy function $\mathcal{H}(x)$ with respect to the state $x$.
It is shown that both the microgrid and the controller dynamics admit a port-Hamiltonian representation which are then interconnected to obtain a closed-loop portHamiltonian system. This allows to easily identify the Hamiltonian energy function and give conditions for stability of the equilibrium state.

In addition, to deal with large signal stability problem of microgrid system, the shifted-Hamiltonian energy function is served as a storage function to ensure incremental-passivity of system (see [88]). Notably, the incremental-passivity of the shifted port-Hamiltonian system is defined as follows:

Definition 2 Consider the port-Hamiltonian system (1). Let $\bar{y}=g^{\top}(\bar{x})(\nabla \mathcal{H}(\bar{x}))$, by defining shifted-Hamiltonian energy function (see [88]) to transfer the state variable $x$ to the desired equilibrium $\bar{x}$ as follows:

$$
\overline{\mathcal{H}}(x):=\mathcal{H}(x)-(\nabla \mathcal{H}(\bar{x}))^{\top}(x-\bar{x})-\mathcal{H}(\bar{x}) .
$$

Then, the port-Hamiltonian system (1) is incremental passive if the mapping $(u-\bar{u}) \rightarrow(y-\bar{y})$ is passive (see also $[88,98,99])$, i.e., there exists a function $\overline{\mathcal{H}}(x)$ such that:

$$
\dot{\overline{\mathcal{H}}}(x)=(\nabla \mathcal{H}(x)-\nabla \mathcal{H}(\bar{x}))^{\top} \dot{x} \leq(u-\bar{u})(y-\bar{y}) .
$$

Where $\bar{x}$ is the desired equilibrium with corresponding control $\bar{u}$.

\section{Microgrid structure and topology}

The microgrid is an emerging concept for an efficient integration of renewable microsource units (see $[1,3$, 100-103] and references herein). An inverter-based $a c$ microgrid consists of microsources (e.g. wind turbine and solar panel equipped with inverters), distribution lines and loads that are connected to main-grid via static switch (common coupling point).

Definition 3 The inverter-based ac microgrid is composed to several units, i.e. microgrid units, including microsource, distribution line and load units (see $[3,90,100]$ and references herein). The microsource unit is referred to unit injecting an amount of power into the microgrid and load unit is also correspond to unit absorbing an amount power from the micro-grid. In addition, distribution unit is referred to unit transferring power within micro-grid. Moreover, the main-grid unit is also corresponded to common coupling port injecting (absorbing) an amount of power into (from) the microgrid (main-grid).

Remark 1 A microgrid topology can be viewed as an unweighted directed graph $\mathcal{G}$ where microgrid units (e.g. main grid, microsource, distribution and load units) correspond to edges and buses correspond to nodes (Fig. 1). Based on the microgrid structure we call a bus: a main-grid bus when a main-grid unit is connected to it; a microsource 
bus when a microsource unit is connected to it; a distribution bus when a distribution unit is connected to it; a load bus when a load unit is connected to it. All buses associate a potential bus and we call a bus a reference bus when all the potential of the buses in the power system are measured with respect to it. The reference bus is assumed to be at ground potential (also see $[90,92,93]$ and references herein).

The inverter-based microgrid model used in this work consists of $g=1$ main-grid bus (in grid-connected mode), $s$ microsource buses, $\ell$ load buses and also one reference bus. Therefore, the total number of buses (nodes or vertices) of the $\mathcal{G}$ microgrid (graph) is $\mathcal{N}=n+1$, with $n=g+s+\ell$. Without loss of generality, it is assumed that the set of nodes (vertices) $\mathcal{N}$ can be partitioned into four ordered subsets called $\mathcal{N}_{G}, \mathcal{N}_{S}, \mathcal{N}_{L}$ and $\mathcal{N}_{0}$ and these subsets are associated to main-grid, microsource, loads nodes and the reference node respectively. We call $\mathcal{V} \in$ $\mathbb{R}^{\mathcal{N}}$ the vector of node potentials. The microgrid graph consists of main-grid, microsource, load and distribution line edges. In other word, there are main-grid edge associated to a main grid unit, microsource edge associated to a microsource unit and also a load edge associated to a load unit and these edges define between main grid node, every microsource node and also every load node and the reference node respectively. Furthermore, distribution edges associated to distribution units that connect main-grid, microsource and load buses Therefore, there are in total $g=1$ main-grid edge (grid-connected mode), $s$ microsource, $\ell$ load and $d$ distribution edges. Hence, there are in total $e=g+s+\ell+d$ edges in micro-grid. Without loss of generality, it is assumed that the set of edges $\mathcal{E}$ can be partitioned into four ordered subsets called $\mathcal{E}_{G}, \mathcal{E}_{S}, \mathcal{E}_{L}$ and $\mathcal{E}_{D}$ associated to main-grid (grid-connected mode), microsource, load and distribution edges respectively. We call $\left(V_{e}, I_{e}\right) \in \mathbb{R}^{e \times e}$ the vectors pair associated to edge voltages and currents respectively. In addition, it is assumed that all microgrid units share a port of the same dimension $p=p_{d q}=2$ in $d q$-form.

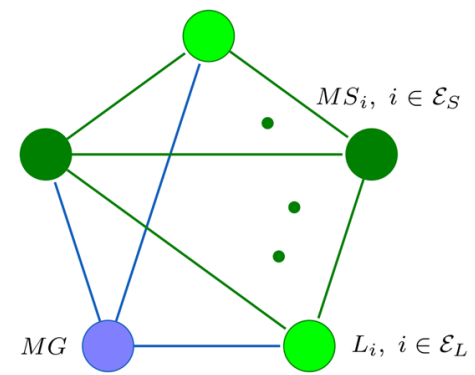

Fig. 1 An inverter-based microgrid topology (MG: main-grid, MS: microsource, L: load)

\section{Inverter-based ac microgrid: port-Hamiltonian formulation}

\subsection{Main-grid Dynamic}

As mentioned in previous section, a main grid unit (grid connected mode) is called a unit injecting (absorbing) an amount of power into (from) the network via common coupling port. In order to describe the dynamic of the main-grid edge $\mathcal{E}_{G}$, the following model is considered:

$$
\begin{aligned}
& \dot{\theta}_{G}=\omega_{G}, \\
& E_{G}=V_{G}, \\
& P_{G}=v_{G}^{\top} i_{G}, \quad Q_{G}=v_{G}^{\top}\left[\begin{array}{cc}
0 & -1 \\
1 & 0
\end{array}\right] i_{G} .
\end{aligned}
$$

Where $\theta_{G}$ and $\omega_{G}$ are phase angle and phase frequency of the main-grid. In addition, $v_{G}$ and $i_{G}$ are voltage and current of main-grid. In addition, $P_{G}$ and $Q_{G}$ are also active and reactive power injected (absorbed) by main grid. It is assumed that in grid-connected mode the frequency and voltage of microgrid are stabilized by main grid. Furthermore, it is also assumed that microgrid can absorb (inject) infinite amount of power from (to) main-grid. Hence, we have:

$$
\begin{aligned}
& 0=-d_{G}\left(\omega_{G}-\bar{\omega}\right)+\left(P_{G}-P_{e}\right), \\
& 0=-a_{G}\left(v_{G}-\bar{V}\right)+\left(Q_{G}-Q_{e}\right) .
\end{aligned}
$$

Where $\bar{V}$ and $\bar{\omega}$ are desired voltage and phase frequency of micro-grid. In addition, $P_{e}$ and $Q_{e}$ are active and reactive power of microgrid and $d_{G}$ and $a_{G}$ are constant coefficients. Furthermore, we have $\omega_{G}=\bar{\omega}$ and $v_{G}=\bar{V}$.

In grid-connected mode, the main grid stabilizes voltage and frequency. In addition, the voltage of main-grid can be considered as follows:

$$
v_{G}=E_{G}\left[\begin{array}{c}
\sin \left(\omega_{G} t\right) \\
\sin \left(\omega_{G} t+\frac{2 \pi}{3}\right) \\
\sin \left(\omega_{G} t-\frac{2 \pi}{3}\right)
\end{array}\right]
$$

In $d q$ form, the voltage of main-grid can also be represented as follows:

$$
v_{G_{d q}}=\sqrt{\frac{2}{3}} E_{G}\left[\begin{array}{l}
\sin \left(\omega_{G} t\right) \\
\cos \left(\omega_{G} t\right)
\end{array}\right]=\bar{v}_{G_{d q}}
$$

Where main-grid connects to microgrid through a port of dimension $p=p_{d q}=2$ in $d q$-form.

\subsection{Microsource dynamics}

As mentioned in Section 3, the microsource unit is called a unit injecting an amount of power to the network. In this section, the dynamic of microsource unit is represented in port-Hamiltonian form. In order to describe this dynamic, a microsource equipped with three-phase inverter is considered (Fig. 2). 
Let $s_{1}, \cdots, s_{6}$ denote the states of the switches $S_{1}, \cdots, S_{6}$ in Fig. 2, where $s=0$ when the switch is open and $s=1$ when the switch is closed. The network switches topology reveals that:

$$
\begin{aligned}
& v_{a o}=v_{d c} s_{1}, v_{a o}=v_{a o}\left(1-s_{4}\right), \\
& v_{b o}=v_{d c} s_{2}, v_{b o}=v_{b o}\left(1-s_{5}\right), \\
& v_{c o}=v_{d c} s_{3}, v_{c o}=v_{c o}\left(1-s_{6}\right) .
\end{aligned}
$$

Considering the constraints imposed by the circuit, i.e., short-cutting the voltage source is not allowed, leads to the observation that the bottom and top switch can never be closed at the same time. Furthermore, for continuity considerations in each phase leg, we have:

$$
s_{1}+s_{4}=1, s_{2}+s_{5}=1, s_{3}+s_{6}=1 \text {. }
$$

The above equations imply that Eq. (7) can be rewritten into a simplified form. Introducing the new variables $s_{a}=$ $s_{1}=s_{4}, s_{b}=s_{2}=s_{5}$ and $s_{c}=s_{3}=s_{6}$ yields the simplified equations:

$$
v_{a o}=v_{d c} s_{a}, v_{b o}=v_{d c} s_{b}, v_{c o}=v_{d c} s_{c} .
$$

Definition 4 The Hamiltonian energy function of the microsource, which denotes the total energy, is given by:

$$
\mathcal{H}\left(\phi, q, q_{d c}\right)=\sum_{j=a, b, c}\left(\frac{1}{2} \frac{\phi_{j}^{2}}{L}+\frac{1}{2} \frac{q_{j}^{2}}{C}\right)+\frac{1}{2} \frac{q_{d c}^{2}}{C_{d c}}
$$

Where $\phi$ is the flux linkage across the inductors, $q$ and $q_{d c}$ the charge in the capacitors. In addition, $L$ and $C$ denote the inductance and capacitance of the microgrid ac-side. In addition $C_{d c}$ denote the capacitance of microsource dcside.
Therefore, the microsource can be represented in portHamiltonian form as follows:

$$
\begin{aligned}
& {\left[\begin{array}{c}
\dot{\phi} \\
\dot{q} \\
\dot{q}_{d c}
\end{array}\right]=\left(\left[\begin{array}{ccc}
\mathbb{O}_{3} & \mathbb{O}_{3} & \widehat{\boldsymbol{s}} \\
\mathbb{O}_{3} & \mathbb{O}_{3} & 0_{31} \\
-\boldsymbol{s}^{\top} & 0_{13} & 0
\end{array}\right]+\left[\begin{array}{ccc}
\mathbb{O}_{3} & -\mathbb{1}_{3} & 0_{31} \\
\mathbb{1}_{3} & \mathbb{O}_{3} & 0_{31} \\
0_{13} & 0_{13} & 0
\end{array}\right]\right.} \\
& \left.-\left[\begin{array}{ccc}
R \rrbracket_{3} & \mathbb{O}_{3} & 0_{31} \\
\mathbb{O}_{3} & \mathbb{O}_{3} & 0_{31} \\
0_{13} & 0_{13} & G_{d c}
\end{array}\right]\right)\left[\begin{array}{c}
\frac{\partial \mathcal{H}}{\partial \phi} \\
\frac{\partial \mathcal{H}}{\partial q} \\
\frac{\partial \mathcal{H}}{\partial q_{d c}}
\end{array}\right]-\left[\begin{array}{c}
\mathbb{0}_{3} \\
\mathbb{\square}_{3} \\
0_{13}
\end{array}\right] i_{S_{a b c}} \\
& +\left[\begin{array}{c}
0_{31} \\
0_{31} \\
1
\end{array}\right] i_{S_{0}} \\
& v_{o, a b c}=\left[\begin{array}{lll}
\mathbb{O}_{3} & \square_{3} & 0_{31}
\end{array}\right] \nabla \mathcal{H}\left(\phi, q, q_{d c}\right) \text {. } \\
& y_{A}=\left[\begin{array}{lll}
\bar{v}_{d c} \rrbracket_{3} & \mathbb{O}_{3}-\bar{i}
\end{array}\right] \nabla \mathcal{H}\left(\phi, q, q_{d c}\right) \text {. }
\end{aligned}
$$

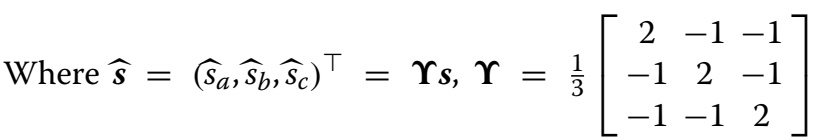
and $s=\left(s_{a}, s_{b}, s_{c}\right)$. In addition, $G_{d c}$ is the conductance of microsource $d c$-side and $x=\left(\phi, q, q_{d c}\right)^{\top}$ is state vector of microsource unit. Furthermore, the $y_{A}$ and $\bar{x}$ are auxiliary output and desired states respectively.

The microsource modeling can be transformed to $d q$ form as follows:

$$
\begin{aligned}
{\left[\begin{array}{l}
\dot{\phi}_{d q} \\
\dot{q}_{d q} \\
\dot{q}_{d c}
\end{array}\right]=} & \left(\left[\begin{array}{ccc}
\mathbb{O}_{2} & \mathbb{O}_{2} & u_{d q} \\
\mathbb{O}_{2} & \mathbb{O}_{2} & 0_{21} \\
-u_{d q}^{\top} & 0_{12} & 0
\end{array}\right]+\left[\begin{array}{ccc}
\bar{\omega} L \mathbf{J} & -\mathbb{D}_{2} & 0_{21} \\
\mathbb{D}_{2} & \bar{\omega} C \mathbf{J} & 0_{21} \\
0_{12} & 0_{12} & 0
\end{array}\right]\right. \\
& \left.-\left[\begin{array}{ccc}
R \mathbb{Q}_{2} & \mathbb{O}_{2} & 0_{21} \\
\mathbb{O}_{2} & \mathbb{O}_{2} & 0_{21} \\
0_{12} & 0_{12} & G_{d c}
\end{array}\right]\right)\left[\begin{array}{c}
\frac{\partial \mathcal{H}}{\partial \phi_{d q}} \\
\frac{\partial \mathcal{H}}{\partial q_{d q}} \\
\frac{\partial \mathcal{H}}{\partial q_{d c}}
\end{array}\right]-\left[\begin{array}{c}
\mathbb{O}_{2} \\
\mathbb{0}_{2} \\
0_{12}
\end{array}\right] i_{S_{d q}}+\left[\begin{array}{c}
0_{21} \\
0_{21} \\
1
\end{array}\right] i_{S_{0}}, \\
v_{o_{d q}}= & {\left[\begin{array}{lll}
\mathbb{O}_{2} & \mathbb{D}_{2} & 0_{21}
\end{array}\right] \nabla \mathcal{H}\left(\phi_{d q}, q_{d q}, q_{d c}\right), } \\
y_{A}= & {\left[\begin{array}{lll}
\bar{v}_{d c} \mathbb{D}_{2} & \mathbb{O}_{2} & -\bar{i}_{d q}
\end{array}\right] \nabla \mathcal{H}\left(\phi_{d q}, q_{d q}, q_{d c}\right) . }
\end{aligned}
$$

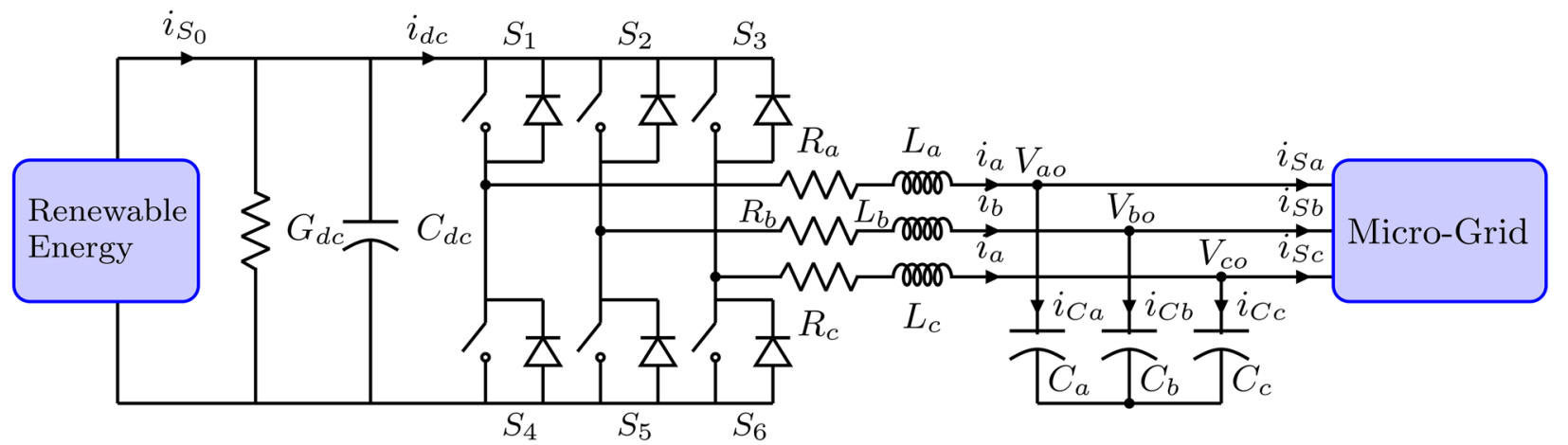

Fig. 2 The microsource equipped with three-phase inverter 
where $\mathbf{J}=\left[\begin{array}{cc}0 & 1 \\ -1 & 0\end{array}\right]$ and also $\Upsilon T_{0 d q}=T_{0 d q}$.

Therefore, the microsource dynamic for $i \in \mathcal{E}_{S}$ can be described as follows:

$$
\begin{aligned}
{\left[\begin{array}{l}
\dot{\phi}_{d q_{i}} \\
\dot{q}_{d q_{i}} \\
\dot{q}_{d c_{i}}
\end{array}\right]=} & \left(\left[\begin{array}{ccc}
\mathbb{O}_{2} & \mathbb{O}_{2} & u_{d q_{i}} \\
\mathbb{O}_{2} & \mathbb{O}_{2} & 0_{21} \\
-u_{d q_{i}}^{\top} & 0_{12} & 0
\end{array}\right]+\left[\begin{array}{ccc}
\bar{\omega} L_{i} \mathbf{J} & -\mathbb{D}_{2} & 0_{21} \\
\mathbb{D}_{2} & \bar{\omega} C_{i} \mathbf{J} & 0_{21} \\
0_{12} & 0_{12} & 0
\end{array}\right]\right. \\
& \left.-\left[\begin{array}{ccc}
R_{i} \mathbb{D}_{2} & \mathbb{O}_{2} & 0_{21} \\
\mathbb{O}_{2} & \mathbb{O}_{2} & 0_{21} \\
0_{12} & 0_{12} & G_{d c_{i}}
\end{array}\right]\right)\left[\begin{array}{c}
\frac{\partial \mathcal{H}_{i}}{\partial \phi_{d q_{i}}} \\
\frac{\partial \mathcal{H}_{i}}{\partial q_{d q_{i}}} \\
\frac{\partial \mathcal{H}}{\partial q_{d c_{i}}}
\end{array}\right]-\left[\begin{array}{c}
\mathbb{O}_{2} \\
\mathbb{0}_{2} \\
0_{12}
\end{array}\right] i_{S_{d q_{i}}}+\left[\begin{array}{c}
0_{21} \\
0_{21} \\
1
\end{array}\right] i_{S_{0 i}}, \\
v_{o_{d q_{i}}} & =\left[\begin{array}{lll}
\mathbb{O}_{2} & \mathbb{O}_{2} & 0_{21}
\end{array}\right] \nabla \mathcal{H}_{S}\left(x_{i}\right), \\
y_{A_{i}}= & {\left[\begin{array}{lll}
\bar{v}_{d c_{i}} \mathbb{D}_{2} & \mathbb{O}_{2} & -\bar{i}_{d q}
\end{array}\right] \nabla \mathcal{H}_{S}\left(x_{i}\right) }
\end{aligned}
$$

Where we have:

$$
\begin{aligned}
& {\left[\begin{array}{c}
\dot{\phi}_{d q_{i}} \\
\dot{q}_{d q_{i}} \\
\dot{q}_{d c_{i}}
\end{array}\right] \in \mathbb{R}^{n_{i}}, u_{d q_{i}} \in \mathbb{R}^{m_{i}}, } \\
v_{o_{d q_{i}}} & \in \mathbb{R}^{p_{i}}, i_{S_{d q_{i}}} \in \mathbb{R}^{p_{i}}, \quad i_{S_{0 i}} \in \mathbb{R} .
\end{aligned}
$$

In addition, $n_{i}=5, m_{i}=2$ and $p_{i}=2$. The portHamiltonian energy function is also defined as $\mathcal{H}_{i}: \mathbb{R}^{n_{i}} \rightarrow$ R.

The aggregated model of the microsource dynamics can be obtained by collecting the port-Hamiltonian forms given by Eq. (12) for $i \in \mathcal{E}_{S}$. Let the numbers:

$$
n_{S}:=\sum_{i=1}^{s} n_{i}, m_{S}:=\sum_{i=1}^{s} m_{i}, p_{S}:=\sum_{i=1}^{s} p_{i}, p_{S_{0}}:=s
$$

the aggregated vectors:

$$
\begin{aligned}
& \boldsymbol{v}_{S, d q}=\operatorname{col}\left(v_{S_{d q_{i}}}\right), \quad \boldsymbol{i}_{S, d q}=\operatorname{col}\left(i_{S_{d q_{i}}}\right) \\
& \boldsymbol{u}_{S, d q}=\operatorname{col}\left(u_{d q_{i}}\right), \quad \boldsymbol{v}_{S, d q}=\operatorname{col}\left(v_{o_{d q_{i}}}\right) \\
& \boldsymbol{v}_{S_{0}}=\operatorname{col}\left(v_{S_{0 i}}\right), \quad \boldsymbol{i}_{S_{0}}=\operatorname{col}\left(i_{S_{0 i}}\right) \\
& {\left[\begin{array}{l}
\boldsymbol{\phi}_{S, d q} \\
\boldsymbol{q}_{S, d q} \\
\boldsymbol{q}_{d c, S}
\end{array}\right]=\operatorname{col}\left[\begin{array}{l}
\boldsymbol{\phi}_{d q_{i}} \\
q_{d q_{i}} \\
q_{d c_{i}}
\end{array}\right],\left[\begin{array}{c}
\frac{\partial \mathcal{H}_{S}}{\partial \boldsymbol{\phi}_{S, d q}} \\
\frac{\partial \mathcal{H}_{S}}{\partial \boldsymbol{q}_{S, d q}} \\
\frac{\partial \mathcal{H}_{S}}{\partial \boldsymbol{q}_{d c, S}}
\end{array}\right]=\operatorname{col}\left[\begin{array}{c}
\frac{\partial \mathcal{H}_{i}}{\partial \phi_{d q_{i}}} \\
\frac{\partial \mathcal{H}_{i}}{\partial q_{d q_{i}}} \\
\frac{\partial \mathcal{H}_{i}}{\partial q_{d c_{i}}}
\end{array}\right]}
\end{aligned}
$$

the interconnection and dissipation matrices:

$$
\begin{gathered}
\mathcal{J}_{S_{u}}:=b d g\left\{\left[\begin{array}{ccc}
\mathbb{O}_{2} & \mathbb{O}_{2} & u_{d q_{i}} \\
\mathbb{O}_{2} & \mathbb{O}_{2} & 0_{21} \\
-u_{d q_{i}}^{\top} & 0_{12} & 0
\end{array}\right]\right\}, \\
\mathcal{J}_{S_{c}}:=b d g\left\{\left[\begin{array}{ccc}
\bar{\omega} L_{i} \mathbf{J} & -\mathbb{\square}_{2} & 0_{21} \\
\mathbb{0}_{2} & \bar{\omega} C_{i} \mathbf{J} & 0_{21} \\
0_{12} & 0_{12} & 0
\end{array}\right]\right\}, \\
\mathcal{R}_{S}:=b d g\left\{\left[\begin{array}{ccc}
R_{i} \mathbb{1}_{2} & \mathbb{O}_{2} & 0_{21} \\
\mathbb{O}_{2} & \mathbb{O}_{2} & 0_{21} \\
0_{12} & 0_{12} & G_{d c_{i}}
\end{array}\right]\right\} .
\end{gathered}
$$

interaction port and microsource port matrices:

$$
\begin{aligned}
& F_{S}:=b d g\left\{\left[\begin{array}{c}
\mathbb{0}_{2} \\
-\mathbb{\square}_{2} \\
0_{12}
\end{array}\right]\right\}, F_{S_{0}}:=b d g\left\{\left[\begin{array}{c}
0_{21} \\
0_{21} \\
1
\end{array}\right]\right\} \\
& A_{S}:=b d g\left\{\left[\begin{array}{c}
\bar{v}_{d c_{i}} \mathbb{\square}_{2} \\
\mathbb{0}_{2} \\
-\bar{i}_{d q_{i}}^{\top}
\end{array}\right]\right\}
\end{aligned}
$$

and the total Hamiltonian function $\mathcal{H}_{S}: \mathbb{R}^{n_{s}} \rightarrow \mathbb{R}$ is defined as follows:

$$
\mathcal{H}_{S}:=\sum_{i=1}^{s} \mathcal{H}_{i}
$$

The aggregated model of the microsource dynamics can be written as:

$$
\begin{aligned}
{\left[\begin{array}{c}
\dot{\boldsymbol{\phi}}_{S, d q} \\
\dot{\boldsymbol{q}}_{S, d q} \\
\dot{\boldsymbol{q}}_{d c, S}
\end{array}\right] } & =\left(\mathcal{J}_{S_{u}}+\mathcal{J}_{S_{c}}-\mathcal{R}_{S}\right)\left[\begin{array}{c}
\frac{\partial \mathcal{H}_{S}}{\partial \boldsymbol{\phi}_{S, d q}} \\
\frac{\partial \mathcal{H}_{S}}{\partial \boldsymbol{q}_{S, d q}} \\
\frac{\partial \mathcal{H}_{S}}{\partial \boldsymbol{q}_{d c, S}}
\end{array}\right]+F_{S} \boldsymbol{i}_{S, d q}+F_{S_{0}} \boldsymbol{i}_{S_{0}}, \\
\boldsymbol{v}_{S, d q} & =F_{S}^{\top} \nabla \mathcal{H}_{S}\left(x_{S}\right), \\
y_{S_{A}} & =A_{S}^{\top}\left(\bar{x}_{S}\right) \nabla \mathcal{H}_{S}\left(x_{S}\right) .
\end{aligned}
$$

Remark 2 The following general port-Hamiltonian form is considered for microsource dynamics:

$$
\left\{\begin{array}{l}
\dot{x}_{S}=\left[\mathcal{J}_{S_{u}}+\mathcal{J}_{S_{c}}-\mathcal{R}_{S}\right] \nabla \mathcal{H}_{S}\left(x_{S}\right)+F_{S} w_{S}+F_{S_{0}} w_{S_{0}}, \\
y_{S_{A}}=A_{S}^{\top}\left(\bar{x}_{S}\right) \nabla \mathcal{H}_{S}\left(x_{S}\right), \\
y_{S}=F_{S}^{\top} \nabla \mathcal{H}_{S}\left(x_{S}\right), \\
y_{S_{0}}=F_{S_{0}}^{\top} \nabla \mathcal{H}_{S}\left(x_{S}\right) .
\end{array}\right.
$$

Where $x_{S}=\left(\boldsymbol{\phi}_{S, d q}, \boldsymbol{q}_{S, d q}, \boldsymbol{q}_{d c, S}\right)$ is state vector of microsource units; $\mathcal{H}_{S}\left(x_{S}\right)$ is Hamiltonian energy function; $\mathcal{J}_{S_{u}}$ and $\mathcal{J}_{S_{c}}$ are interconnection matrices and $\mathcal{R}_{\mathcal{S}}$ is dissipation matrices; $\left(w_{S}, y_{S}\right)=\left(\boldsymbol{i}_{S, d q}, \boldsymbol{v}_{S, d q}\right)$ is conjugated interaction port variables; $\left(w_{S_{0}}, y_{S_{0}}\right)=\left(\boldsymbol{i}_{S_{0}}, \boldsymbol{v}_{d c, S}\right)$ is also conjugated microsource port variables. Other matrices can be defined as follows, $F_{S}$ interaction port matrix and $F_{S_{0}}$ microsource port matrix. Furthermore, the $y_{S_{A}}$ is auxiliary passive outputs. 


\subsection{Distribution line dynamics}

A distribution line unit is called a unit that transfer power in the microgrid and also absorb or inject a little amount of power compared to microsource and load units. It is assumed that all distribution line units are describing by mixed lines (i.e. $R-L$ series). A circuit representation of series line is illustrated in Fig. 3. The model consists of an $R-L$ unit (resistive-inductive line). Therefore, The model of the distribution line unit is simply given by the following port-Hamiltonian formulation:

$$
\begin{aligned}
\dot{\phi} & =-\left[R \otimes \mathbb{1}_{3}\right] \nabla \mathcal{H}(\phi)+v, \\
i & =\nabla \mathcal{H}(\phi) .
\end{aligned}
$$

Where $\phi$ is the magnetic flux in the inductor.

Definition 5 The Hamiltonian energy function of the distribution line, $\mathcal{H}(\phi): \mathbb{R}^{3} \rightarrow \mathbb{R}$, is described as follows:

$$
\mathcal{H}(\phi):=\frac{1}{2} \phi^{\top}\left(L \otimes \mathbb{1}_{3}\right)^{-1} \phi .
$$

In order to describe the port-Hamiltonian model in $d q$ form, we have:

$$
\begin{aligned}
\dot{\phi}_{d q} & =\left[\bar{\omega} L \mathbf{J}-R \rrbracket_{2}\right] \frac{\partial \mathcal{H}\left(\phi_{d q}\right)}{\partial \phi_{d q}}+v_{d q}, \\
i_{d q} & =\frac{\partial \mathcal{H}\left(\phi_{d q}\right)}{\partial \phi_{d q}} .
\end{aligned}
$$

Where $\mathbf{J}=\left[\begin{array}{cc}0 & 1 \\ -1 & 0\end{array}\right]$. In addition, $\mathcal{H}\left(\phi_{d q}\right): \mathbb{R}^{2} \rightarrow \mathbb{R}$ is Hamiltonian energy function and described as follows:

$$
\mathcal{H}\left(\phi_{d q}\right):=\frac{1}{2} \phi_{d q}^{\top}\left(L \otimes \mathbb{I}_{2}\right)^{-1} \phi_{d q} .
$$

Therefore, the distribution line dynamic for $i \in \mathcal{E}_{D}$ can be described as follows:

$$
\begin{aligned}
\dot{\phi}_{d q_{i}} & =\left[\bar{\omega} L_{i} \mathbf{J}-R_{i} \mathbb{1}_{2}\right] \frac{\partial \mathcal{H}\left(\phi_{d q_{i}}\right)}{\partial \phi_{d q_{i}}}+v_{d q_{i}}, \\
i_{d q_{i}} & =\frac{\partial \mathcal{H}\left(\phi_{d q_{i}}\right)}{\partial \phi_{d q_{i}}} .
\end{aligned}
$$

Where we have:

$$
\phi_{d q_{i}} \in \mathbb{R}^{n_{i}}, \quad v_{d q_{i}} \in \mathbb{R}^{p_{i}}, \quad i_{d q_{i}} \in \mathbb{R}^{p_{i}} .
$$

Where $n_{i}=2$ and $p_{i}=2$. In addition, port-Hamiltonian energy function defines as $\mathcal{H}_{i}\left(\phi_{d q_{i}}\right): \mathbb{R}^{n_{i}} \rightarrow \mathbb{R}$.

The aggregated model of the distribution line dynamics can be obtained by collecting the port-Hamiltonian forms given by Eq. (25) for $i \in \mathcal{E}_{D}$. Let the numbers:

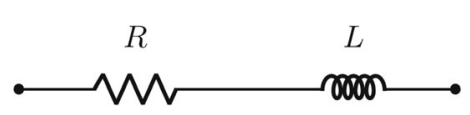

Fig. $3 R-L$ model of a distribution unit

$$
n_{D}:=\sum_{i=1}^{d} n_{i}, \quad, \quad p_{D}:=\sum_{i=1}^{d} p_{i}
$$

the aggregated vectors:

$$
\begin{aligned}
\boldsymbol{i}_{D, d q} & =\operatorname{col}\left(i_{d q_{i}}\right), \boldsymbol{\nu}_{D, d q}=\operatorname{col}\left(v_{d q_{i}}\right) \\
\boldsymbol{\phi}_{D, d q} & =\operatorname{col}\left(\phi_{d q_{i}}\right), \frac{\partial \mathcal{H}_{D}}{\partial \boldsymbol{\phi}_{D, d q}}=\operatorname{col}\left(\frac{\partial \mathcal{H}_{i}}{\partial \phi_{d q_{i}}}\right) .
\end{aligned}
$$

the interconnection and dissipation matrix:

$$
\begin{aligned}
& \mathcal{J}_{D_{c}}:=b d g\left\{\bar{\omega} L_{i} \mathbf{J}\right\}, \\
& \mathcal{R}_{D}:=b d g\left\{R_{i} \rrbracket_{2}\right\} .
\end{aligned}
$$

interaction port matrices:

$$
F_{D}:=b d g\left\{\rrbracket_{2}\right\}
$$

and the total Hamiltonian energy function $\mathcal{H}_{D}: \mathbb{R}^{n_{D}} \rightarrow \mathbb{R}$ :

$$
\mathcal{H}_{D}:=\sum_{i=1}^{d} \mathcal{H}\left(\phi_{d q_{i}}\right)
$$

The aggregated model of the distribution line edges can be written as:

$$
\begin{aligned}
\dot{\boldsymbol{\phi}}_{D, d q} & =\left(\mathcal{J}_{D_{c}}-\mathcal{R}_{D}\right) \frac{\partial \mathcal{H}_{D}}{\partial \boldsymbol{\phi}_{D, d q}}+F_{D} \boldsymbol{\nu}_{D, d q}, \\
\boldsymbol{i}_{D, d q} & =F_{D}^{\top} \frac{\partial \mathcal{H}_{D}}{\partial \boldsymbol{\phi}_{D, d q}} .
\end{aligned}
$$

Remark 3 The general port-Hamiltonian formulation of the distribution line dynamics is given by:

$$
\left\{\begin{array}{c}
\dot{x}_{D}=\left[\mathcal{J}_{D_{c}}-\mathcal{R}_{D}\right] \nabla \mathcal{H}_{D}\left(x_{D}\right)+F_{D} w_{D} \\
y_{D}=F_{D}^{\top} \nabla \mathcal{H}_{D}\left(x_{D}\right)
\end{array}\right.
$$

\subsection{Load dynamics}

A load unit is called a power unit that absorbs an amount of power from the micro-grid. Without loss of generality, it is assumed that all load units are describing by $R-C$ parallels. A circuit representation of load unit (resistance-capacitance parts)is illustrated in Fig. 4 . The model of the load unit is simply given by the following port-Hamiltonian system:

$$
\begin{aligned}
& \dot{q}=-\left[R \otimes \square_{3}\right] \nabla \mathcal{H}(q)+i, \\
& v=\nabla \mathcal{H}(q) .
\end{aligned}
$$

Where $q$ is the charge in the capacitor. 


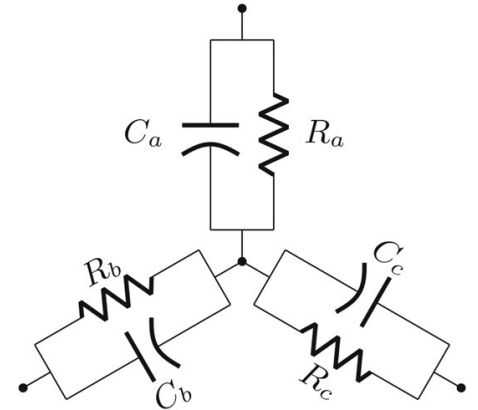

Fig. $4 R-C$ model of a load unit

Definition 6 The Hamiltonian energy function of load unit, $\mathcal{H}(q): \mathbb{R}^{3} \rightarrow \mathbb{R}$, is described as follows:

$$
\mathcal{H}(q):=\frac{1}{2} q^{\top}\left(C \otimes \mathbb{I}_{3}\right)^{-1} q
$$

In order to describe the port-Hamiltonian model in $d q$ form, we have:

$$
\begin{aligned}
\dot{q}_{d q} & =\left[\bar{\omega} C \mathbf{J}-R \rrbracket_{2}\right] \frac{\partial \mathcal{H}\left(q_{d q}\right)}{\partial q_{d q}}+i_{d q} \\
v_{d q} & =\frac{\partial \mathcal{H}\left(q_{d q}\right)}{\partial q_{d q}} .
\end{aligned}
$$

Where $\mathbf{J}=\left[\begin{array}{cc}0 & 1 \\ -1 & 0\end{array}\right]$. In addition, $\mathcal{H}\left(q_{d q}\right): \mathbb{R}^{2} \rightarrow \mathbb{R}$ is Hamiltonian energy function and described as follows:

$$
\mathcal{H}\left(q_{d q}\right):=\frac{1}{2} q_{d q}^{\top}\left(C \otimes \mathbb{I}_{2}\right)^{-1} q_{d q}
$$

Therefore, the load dynamic for $i \in \mathcal{E}_{L}$ can be described as follows:

$$
\begin{aligned}
\dot{q}_{d q_{i}} & =\left[\bar{\omega} C_{i} \mathbf{J}-R_{i} \mathbb{\square}_{2}\right] \frac{\partial \mathcal{H}\left(q_{d q_{i}}\right)}{\partial q_{d q_{i}}}+i_{d q_{i}}, \\
v_{d q_{i}} & =\frac{\partial \mathcal{H}\left(q_{d q_{i}}\right)}{\partial q_{d q_{i}}} .
\end{aligned}
$$

Where we have:

$$
q_{d q_{i}} \in \mathbb{R}^{n_{i}}, \quad i_{d q_{i}} \in \mathbb{R}^{p_{i}}, \quad v_{d q_{i}} \in \mathbb{R}^{p_{i}}
$$

In addition, $n_{i}=2$ and $p_{i}=2$. The port-Hamiltonian energy function is also defined as $\mathcal{H}_{i}\left(q_{d q_{i}}\right): \mathbb{R}^{n_{i}} \rightarrow \mathbb{R}$.

The aggregated model of the load dynamics are obtained by collecting the port-Hamiltonian forms given by Eq. (38) for $i \in \mathcal{E}_{L}$. Let the numbers:

$$
n_{L}:=\sum_{i=1}^{\ell} n_{i}, \quad, \quad p_{L}:=\sum_{i=1}^{\ell} p_{i} .
$$

the aggregated vectors:

$$
\begin{aligned}
& \boldsymbol{i}_{L, d q}=\operatorname{col}\left(i_{d q_{i}}\right), \boldsymbol{v}_{L, d q}=\operatorname{col}\left(v_{d q_{i}}\right), \\
& \boldsymbol{q}_{L, d q}=\operatorname{col}\left(q_{d q_{i}}\right), \frac{\partial \mathcal{H}_{L}}{\partial \boldsymbol{q}_{L, d q}}=\operatorname{col}\left(\frac{\partial \mathcal{H}_{i}}{\partial q_{d q_{i}}}\right),
\end{aligned}
$$

the interconnection and dissipation matrix:

$$
\begin{aligned}
& \mathcal{J}_{L_{c}}:=b d g\left\{\bar{\omega} C_{i} \mathbf{J}\right\}, \\
& \mathcal{R}_{L}:=b d g\left\{R_{i} \rrbracket_{2}\right\} .
\end{aligned}
$$

interaction port matrices:

$$
F_{L}:=b d g\left\{\mathbb{D}_{2}\right\}
$$

and the total Hamiltonian function $\mathcal{H}_{L}: \mathbb{R}^{n_{L}} \rightarrow \mathbb{R}$ for load dynamics:

$$
\mathcal{H}_{L}:=\sum_{i=1}^{\ell} \mathcal{H}\left(\phi_{d q_{i}}\right) .
$$

The aggregated model of the distribution load dynamics can be written as:

$$
\begin{aligned}
\dot{\boldsymbol{q}}_{L, d q} & =\left(\mathcal{J}_{L_{c}}-\mathcal{R}_{L}\right) \frac{\partial \mathcal{H}_{L}}{\partial \boldsymbol{q}_{L, d q}}+F_{L} i_{L, d q}, \\
\boldsymbol{v}_{L, d q} & =F_{L}^{\top} \frac{\partial \mathcal{H}_{L}}{\partial \boldsymbol{q}_{L, d q}} .
\end{aligned}
$$

Remark 4 The general port-Hamiltonian formulation of the distribution load dynamics is given by:

$$
\left\{\begin{array}{c}
\dot{x}_{L}=\left[\mathcal{J}_{L_{c}}-\mathcal{R}_{L}\right] \nabla \mathcal{H}_{L}\left(x_{L}\right)+F_{L} w_{L}, \\
y_{L}=F_{L}^{\top} \nabla \mathcal{H}_{L}\left(x_{L}\right) .
\end{array}\right.
$$

\subsection{Microgrid overall dynamic}

The microgrid overall dynamic is obtained by collecting the aggregated unit dynamics Eqs. (3), (5), (20), (33) and (46). Therefore, the overall dynamic can be rewritten in port-Hamiltonian formulation as follows (Fig. 5):

$$
\begin{aligned}
0 & =w_{G}-\bar{w}_{G}=i_{G_{d q}}-\bar{i}_{G_{d q}}, \\
\dot{x}_{S} & =\left[\mathcal{J}_{S_{u}}+\mathcal{J}_{S_{c}}-\mathcal{R}_{S}\right] \nabla \mathcal{H}_{S}\left(x_{S}\right)+F_{S} w_{S}+F_{S_{0}} w_{S_{0}}, \\
\dot{x}_{L} & =\left[\mathcal{J}_{L_{c}}-\mathcal{R}_{L}\right] \nabla \mathcal{H}_{L}\left(x_{L}\right)+F_{L} w_{L}, \\
\dot{x}_{D} & =\left[\mathcal{J}_{D_{c}}-\mathcal{R}_{D}\right] \nabla \mathcal{H}_{D}\left(x_{D}\right)+F_{D} w_{D}, \\
y_{G} & =\bar{y}_{G}=\bar{v}_{G_{d q}}, \\
y_{S_{A}} & =A_{S}^{\top}\left(\bar{x}_{S}\right) \nabla \mathcal{H}_{S}(x), \\
y_{S} & =F_{S}^{\top} \nabla \mathcal{H}_{S}\left(x_{S}\right) \\
y_{L} & =F_{L}^{\top} \nabla \mathcal{H}_{L}\left(x_{L}\right) \\
y_{D} & =F_{D}^{\top} \nabla \mathcal{H}_{D}\left(x_{D}\right), \\
y_{S_{0}} & =F_{S_{0}}^{\top} \nabla \mathcal{H}_{S}\left(x_{S}\right) .
\end{aligned}
$$


Load and distribution line dynamics

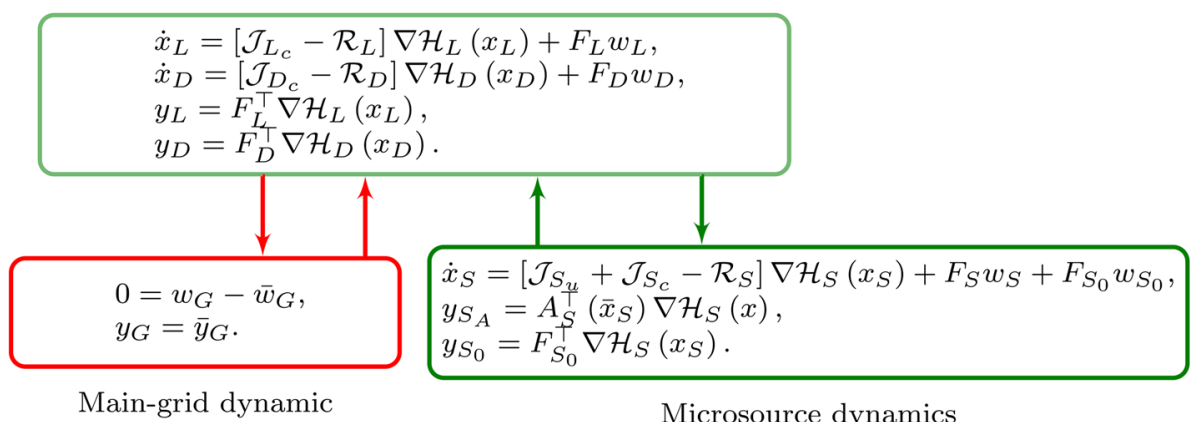

Microsource dynamics

Fig. 5 Microgrid Overall Dynamic: port-Hamiltonian formulation

Let collecting the numbers (14), (27) and (40) as follows:

$$
\begin{aligned}
& n_{T}:=n_{S}+n_{L}+n_{D}, m_{T}:=m_{S}, \\
& p_{T}:=p_{S}+p_{L}+p_{D}, p_{T_{0}}:=p_{S_{0}},
\end{aligned}
$$

Collecting interconnection matrices of the microgrid unit dynamics (16), (29) and (42):

$$
\begin{aligned}
& \mathcal{J}_{T_{u}}:=b d g\left\{\mathcal{J}_{S_{u}}, 0,0\right\}, \mathcal{J}_{T_{c}}:=b d g\left\{\mathcal{J}_{S_{c}}, \mathcal{J}_{L_{c}}, \mathcal{J}_{D_{c}}\right\}, \\
& \mathcal{J}_{S_{u}}:=b d g\left\{\left[\begin{array}{ccc}
\mathbb{O}_{2} & \mathbb{O}_{2} & u_{d q_{i}} \\
\mathbb{O}_{2} & \mathbb{O}_{2} & 0_{21} \\
-u_{d q_{i}}^{\top} & 0_{12} & 0
\end{array}\right]\right\}, \\
& \mathcal{J}_{S_{c}}:=b d g\left\{\left[\begin{array}{ccc}
\bar{\omega} L_{i} \mathbf{J} & -\mathbb{\square}_{2} & 0_{21} \\
\mathbb{1}_{2} & \bar{\omega} C_{i} \mathbf{J} & 0_{21} \\
0_{12} & 0_{12} & 0
\end{array}\right]\right\}, \\
& \mathcal{J}_{L_{c}}:=b d g\left\{\bar{\omega} C_{i} \mathbf{J}\right\}, \mathcal{J}_{D_{c}}:=b d g\left\{\bar{\omega} L_{i} \mathbf{J}\right\} .
\end{aligned}
$$

Collecting dissipation matrices of the microgrid unit dynamics (16), (29) and (42):

$$
\begin{aligned}
& \mathcal{R}_{T}:=b d g\left\{\mathcal{R}_{S}, \mathcal{R}_{L}, \mathcal{R}_{D}\right\}, \\
& \mathcal{R}_{S}:=b d g\left\{\left[\begin{array}{ccc}
R_{i} \rrbracket_{2} & \mathbb{O}_{2} & 0_{21} \\
\mathbb{O}_{2} & 0_{2} & 0_{21} \\
0_{12} & 0_{12} & G_{d c_{i}}
\end{array}\right]\right\}, \\
& \mathcal{R}_{L}:=b d g\left\{R_{i} \rrbracket_{2}\right\}, \mathcal{R}_{D}:=b d g\left\{R_{i} \rrbracket_{2}\right\} .
\end{aligned}
$$

and collecting interaction port matrices of the microgrid unit dynamics (17), (30) and (43):

$$
\begin{aligned}
& F_{T}:=b d g\left\{F_{G}, F_{S}, 0, F_{D}\right\}, \\
& F_{G}:=1, F_{S}:=b d g\left\{\left[\begin{array}{c}
\mathbb{0}_{2} \\
-\rrbracket_{2} \\
0_{12}
\end{array}\right]\right\}, \\
& F_{L}:=b d g\left\{\square_{2}\right\}, F_{D}:=b d g\left\{\square_{2}\right\} .
\end{aligned}
$$

and collecting microsource port matrices (17):

$$
F_{T_{0}}:=b d g\left\{F_{S_{0}}, 0,0\right\} ; F_{S_{0}}:=b d g\left\{\left[\begin{array}{c}
0_{21} \\
0_{21} \\
1
\end{array}\right]\right\} \text {. }
$$

and collecting overall auxiliary passive output matrices (19):

$$
A_{T}:=b d g\left\{A_{S}, 0,0\right\} ; A_{S}:=b d g\left\{\left[\begin{array}{c}
\bar{v}_{d c_{i}}^{\mathbb{1}_{2}} \\
\mathbb{0}_{2} \\
-\bar{i}_{S_{d q_{i}}^{\top}}^{\top}
\end{array}\right]\right\} \text {. }
$$

The overall port-Hamiltonian form of inverter-based microgrid can be represented as follows:

$$
\begin{aligned}
0= & w_{G}-\bar{w}_{G}, \\
\dot{x}_{T}= & {\left[\mathcal{J}_{T_{u}}+\mathcal{J}_{T_{c}}-\mathcal{R}_{T}\right] \nabla \mathcal{H}_{T}\left(x_{T}\right) } \\
& +F_{T} w_{T}+F_{T_{0}} w_{T_{0}}, \\
y_{G}= & \bar{y}_{G}, \\
y_{T_{A}}= & A_{T}^{\top}\left(\bar{x}_{T}\right) \nabla \mathcal{H}_{T}\left(x_{T}\right), \\
y_{T}= & F_{T}^{\top} \nabla \mathcal{H}_{T}\left(x_{T}\right), \\
y_{T_{0}}= & F_{T_{0}}^{\top} \nabla \mathcal{H}_{T}\left(x_{T}\right) .
\end{aligned}
$$

Where the overall Hamiltonian energy function $\mathcal{H}_{T}$ : $\mathbb{R}^{n_{T}} \rightarrow \mathbb{R}$ is given by:

$$
\mathcal{H}_{T}:=\mathcal{H}_{G}+\mathcal{H}_{L}+\mathcal{H}_{D}
$$

Furthermore, in overall microgrid dynamic (54), the state space vectors are defined as follows:

$\begin{aligned} x_{T} & =\left[\begin{array}{l}\boldsymbol{\phi}_{S, d q} \\ \boldsymbol{q}_{S, d q} \\ \boldsymbol{q}_{d c, S} \\ \boldsymbol{q}_{L, d q} \\ \boldsymbol{\phi}_{D, d q}\end{array}\right] \in \mathbb{R}^{n_{T}}, w_{T}=\left[\begin{array}{c}\boldsymbol{i}_{S, d q} \\ \boldsymbol{i}_{L, d q} \\ \boldsymbol{v}_{D, d q}\end{array}\right] \in \mathbb{R}^{p_{T},} \\ y_{T} & =\left[\begin{array}{c}\boldsymbol{v}_{S, d q} \\ \boldsymbol{v}_{L, d q} \\ \boldsymbol{i}_{D, d q}\end{array}\right] \in \mathbb{R}^{p_{T}}, w_{T_{0}}=\boldsymbol{i}_{S_{0}} \in \mathbb{R}^{p_{T_{0}}}, y_{T_{0}}=\boldsymbol{v}_{S_{0}} \in \mathbb{R}^{p_{T_{0}}},\end{aligned}$

As mentioned in Section 3, the microgrid topology can be define based on graph $\mathcal{G}$. In addition, the incidence matrix $D \in \mathbb{R} \bigvee \times e$ of the microgrid graph is obtained by treating buses as nodes and power units (main-grid, microsource, distribution lines and loads) as edges and given by: 


$$
D=\left[\begin{array}{ccccc}
\hat{\sigma} \rrbracket_{G} & 0 & 0 & 0 & \hat{\sigma} D_{G} \\
0 & \rrbracket_{S_{V}} & 0 & 0 & D_{S_{V}} \\
0 & 0 & \rrbracket_{S_{F}} & 0 & D_{S_{F}} \\
0 & 0 & 0 & \square_{L} & D_{L} \\
-\hat{\sigma} \mathbf{1}_{G}^{\top} & -\mathbf{1}_{S_{V}}^{\top} & -\mathbf{1}_{S_{F}}^{\top} & -\mathbf{1}_{L}^{\top} & 0
\end{array}\right]
$$

Where $\hat{\sigma}=(1-\sigma)$ and $\sigma$ shows the microgrid different operation modes, i.e. $\sigma=0$ for grid-connected and $\sigma=$ 1 for islanding mode. In addition, $\mathbf{1}_{\times}$is correspond to a column vector with all its entries equal to one and the of size of vectors defines as follows:

$$
\mathbf{1}_{G} \in \mathbb{R}^{g},\left[\begin{array}{ll}
\mathbf{1}_{S_{V}} & \mathbf{1}_{S_{F}}
\end{array}\right] \in \mathbb{R}^{s}, \mathbf{1}_{L} \in \mathbb{R}^{\ell}
$$

Notably, in islanding mode, the microsources are divided to grid-forming (stabilizing frequency) and grid feeding (stabilizing voltage and power). Hence, the sub-matrices, $D_{S_{V}}$ and $D_{S_{F}}$, are refereed to these divisions. Furthermore, the sub-matrix $D^{\prime}$ represents the incidence matrix of the $\mathcal{G}^{\prime}$ microgrid graph, that is obtained by eliminating the reference node and edges that are connected to it.

$$
D^{\prime \top}=\left[\begin{array}{llll}
\hat{\sigma} D_{G} & D_{S_{V}} & D_{S_{F}} & D_{L}
\end{array}\right]
$$

In addition, the sub-matrix of microsource incidence matrix is also decomposed to capture the information about the microgrid different operation modes (gridconnected and islanding).

In this section, the vector of node voltages $\mathcal{V}$ and the vector of edge voltages and currents $\left(V_{e}, I_{e}\right)$ are defined as follows:

$$
\mathcal{V}:=\left[\begin{array}{c}
\mathcal{V}_{G} \\
\mathcal{V}_{S_{V}} \\
\mathcal{V}_{S_{F}} \\
\mathcal{V}_{L} \\
0
\end{array}\right] \in \mathbb{R} V, V_{e}:=\left[\begin{array}{c}
v_{G} \\
v_{S_{V}} \\
v_{S_{F}} \\
v_{L} \\
v_{D}
\end{array}\right] \in \mathbb{R}^{e}, I_{e}:=\left[\begin{array}{c}
i_{G} \\
i_{S_{V}} \\
i_{S_{F}} \\
i_{L} \\
i_{D}
\end{array}\right] \in \mathbb{R}^{e}
$$

Where the reference node is considered to be a ground potential.

Using Kirchhoff's current and voltage laws we get then:

$$
\mathbf{0}_{\bigvee}=D I_{e}, \quad V_{e}=D^{\top} \mathcal{V}
$$

Then, recalling the definition of incidence matrix given in (57), we have

$$
\begin{aligned}
& \left\{\begin{array}{l}
0=i_{G}+\hat{\sigma} D_{G} i_{D}, \\
0=i_{S_{V}}+D_{S_{V}} i_{D} \\
0=i_{S_{F}}+D_{S_{F}} i_{D} \\
0=i_{L}+D_{L} i_{D} \\
0=\hat{\sigma} \mathbf{1}_{G}^{\top} i_{G}+\mathbf{1}_{S_{V}}^{\top} i_{S_{V}}+\mathbf{1}_{S_{F}}^{\top} i_{S_{F}}+\mathbf{1}_{L}^{\top} i_{L} .
\end{array}\right. \\
& \left\{\begin{array}{l}
v_{G}=\mathcal{V}_{G}, \\
v_{S_{V}}=\mathcal{V}_{S_{V}}, \\
v_{S_{F}}=\mathcal{V}_{S_{F}}, \\
v_{L}=\mathcal{V}_{L}, \\
v_{D}=D_{G}^{\top} \mathcal{V}_{G}+D_{S_{V}}^{\top} \mathcal{V}_{S_{V}}+D_{S_{F}}^{\top} \mathcal{V}_{S_{F}}+D_{L}^{\top} \mathcal{V}_{L} .
\end{array}\right.
\end{aligned}
$$

Therefore, the overall interconnection law is obtained as follows:

$$
w=\mathcal{T} y
$$

By defining $w:=\operatorname{col}\left(i_{G}, i_{S_{F}}, i_{S_{V}}, i_{L}, v_{D}\right)$ and $y:=$ $\operatorname{col}\left(v_{G}, v_{S_{F}}, v_{S_{V}}, v_{L}, i_{D}\right)$, the overall interconnection matrix is obtained as follows:

$$
\mathcal{T}=\left[\begin{array}{ccccc}
0 & 0 & 0 & 0 & -\hat{\sigma} D_{G} \\
0 & 0 & 0 & 0 & -D_{S_{V}} \\
0 & 0 & 0 & 0 & -D_{S_{F}} \\
0 & 0 & 0 & 0 & -D_{L} \\
\hat{\sigma} D_{G}^{\top} & D_{S_{V}}^{\top} & D_{S_{F}}^{\top} & D_{L}^{\top} & 0
\end{array}\right]
$$

Note that the overall interconnection law is power preserving. In fact

$$
w^{\top} y=y^{\top} \mathcal{T}^{\top} y=0,
$$

Where the matrix $\mathcal{T}$ is skew-symmetry. We also have:

$$
w_{T}=\mathcal{T}_{T} y_{T}+\left[\begin{array}{c}
0 \\
0 \\
0 \\
\hat{\sigma} D_{G}^{\top}
\end{array}\right] v_{G}=\mathcal{T}_{T} y_{T}+G_{T} \bar{y}_{G},
$$

where the $v_{G}$ is the voltage of main-grid. Therefore the matrix $\mathcal{T}_{T}$ is defined as follows:

$$
\mathcal{T}_{T}=\left[\begin{array}{cccc}
0 & 0 & 0 & -D_{S_{V}} \\
0 & 0 & 0 & -D_{S_{F}} \\
0 & 0 & 0 & -D_{L} \\
D_{S_{V}}^{\top} & D_{S_{F}}^{\top} & D_{L}^{\top} & 0
\end{array}\right]
$$

Remark 5 By adding the dynamic of main-grid in gridconnected mode (3) to overall port-Hamiltonian modeling (54) and using the interconnection law (68), we then have:

$$
\begin{aligned}
0= & w_{G}-\bar{w}_{G} \\
\dot{x}_{T}= & {\left[\mathcal{J}_{T_{u}}+\mathcal{J}_{T_{c}}-\mathcal{R}_{T}+F_{T} \mathcal{T}_{T} F_{T}^{\top}\right] \nabla \mathcal{H}_{T}\left(x_{T}\right) } \\
& +G_{T} \bar{y}_{G}+F_{T_{0}} w_{T_{0}}, \\
y_{T_{A}}= & A_{T}^{\top}\left(\bar{x}_{T}\right) \nabla \mathcal{H}_{T}\left(x_{T}\right) \\
y_{T}= & F_{T}^{\top} \nabla \mathcal{H}_{T}\left(x_{T}\right) \\
y_{G}= & \bar{y}_{G} \\
y_{T_{0}}= & F_{T_{0}}^{\top} \nabla \mathcal{H}_{T}\left(x_{T}\right) .
\end{aligned}
$$

Where the matrix $F_{T} \mathcal{T} F_{T}^{\top}$ is skew-symmetry.

In addition, by using $i_{G}=\nabla \mathcal{H}_{T}\left(x_{D}\right)$, so we have:

$$
\begin{aligned}
0=w_{G}-\bar{w}_{G} & =-\hat{\sigma} D_{G} i_{D}+\bar{w}_{G} \\
& =-\hat{\sigma} D_{G} \nabla \mathcal{H}_{T}\left(x_{D}\right)+\bar{w}_{G} .
\end{aligned}
$$


Remark 6 The microgrid overall port-Hamiltonian system (69) satisfies the power balance equation:

$$
\begin{aligned}
\dot{\mathcal{H}}_{T} & =\left[\nabla \mathcal{H}_{T}\left(x_{T}\right)\right]^{\top} \dot{x}_{T} \\
& =-\left[\nabla \mathcal{H}_{T}\left(x_{T}\right)\right]^{\top} \mathcal{R}_{T}\left[\nabla \mathcal{H}_{T}\left(x_{T}\right)\right] \\
& +\left[\nabla \mathcal{H}_{T}\left(x_{T}\right)\right]^{\top}\left\{G_{T} \bar{y}_{G}+F_{T_{0}} w_{T_{0}}\right\} \\
& \leq\left[\nabla \mathcal{H}_{T}\left(x_{T}\right)\right]^{\top}\left\{G_{T} \bar{y}_{G}\right\}+\left[\nabla \mathcal{H}_{T}\left(x_{T}\right)\right]^{\top}\left\{F_{T_{0}} w_{T_{0}}\right\} \\
& =w_{G}^{\top} \bar{y}_{G}+y_{T_{0}}^{\top} w_{T_{0}}
\end{aligned}
$$

Therefore, the overall port-Hamiltonian system verifies the dissipation inequality with Hamiltonian storage function $\mathcal{H}_{T}$. Where the term:

- $\dot{\mathcal{H}}_{T}$ accounts for the stored power (difference between supplied and absorbed power) in micro-grid;

- $\left[\nabla \mathcal{H}_{T}\left(x_{T}\right)\right]^{\top} \mathcal{R}_{T}\left[\nabla \mathcal{H}_{T}\left(x_{T}\right)\right]$ represents the dissipated power in micro-grid;

- $w_{G}^{\top} \bar{y}_{G}+y_{T_{0}}^{\top} w_{T_{0}}$ represents the supplied power in micro-grid.

\section{Microgrid open-loop analysis}

The set of assignable equilibrium to the microgrid overall dynamic (69) is given by:

$$
\begin{aligned}
0 & =\bar{w}_{G}-\bar{w}_{G}, \\
0 & =\left[\mathcal{J}_{T_{u}}(\bar{u})+\mathcal{J}_{T_{c}}-\mathcal{R}_{T}+F_{T} \mathcal{T}_{T} F_{T}^{\top}\right] \nabla \mathcal{H}_{T}\left(\bar{x}_{T}\right) \\
& +G_{T} \bar{y}_{G}+F_{T_{0}} w_{T_{0}}, \\
\bar{y}_{T_{A}} & =A_{T}^{\top}\left(\bar{x}_{T}\right) \nabla \mathcal{H}_{T}\left(\bar{x}_{T}\right), \\
\bar{y}_{T} & =F_{T}^{\top} \nabla \mathcal{H}_{T}\left(\bar{x}_{T}\right) \\
\bar{y}_{G} & =\bar{y}_{G} \\
\bar{y}_{T_{0}} & =F_{T_{0}}^{\top} \nabla \mathcal{H}_{T}\left(\bar{x}_{T}\right),
\end{aligned}
$$

Where $\bar{x}_{T} \in \mathbb{R}^{n_{T}}$ is the desired equilibrium with corresponding control $\bar{u}$.

Proposition 1 Suppose now that there exists an equilibrium $\bar{x}_{T}$. Then, the shifted Hamiltonian energy function (2) transfers the state variable $x_{T}$ to the desired equilibrium $\bar{x}_{T}$.

$$
\overline{\mathcal{H}}_{T}(s):=\mathcal{H}_{T}(s)-\left(\nabla \mathcal{H}_{T}(\bar{s})\right)^{\top}(s-\bar{s})-\mathcal{H}_{T}(\bar{s}) .
$$

Proof By defining $z:=\nabla \mathcal{H}_{T}(s), \bar{z}:=\nabla \mathcal{H}_{T}(\bar{s}), \lambda:=$ $\nabla \mathcal{H}_{S}(s)$ and $\bar{\lambda}:=\nabla \mathcal{H}_{S}(\bar{s})$ and recalling the equations (69), we have

$$
\begin{aligned}
& \dot{\overline{\mathcal{H}}}_{T}(s)=\left(\nabla \mathcal{H}_{T}(s)-\nabla \mathcal{H}_{T}(\bar{s})\right)^{\top} \dot{s} \\
&=(z-\bar{z})^{\top}\left\{\left[\mathcal{J}_{T_{u}}+\mathcal{J}_{T_{c}}-\mathcal{R}_{T}+F_{T} \mathcal{T}_{T} F_{T}^{\top}\right] z\right. \\
&\left.\quad+G_{T} \bar{y}_{G}+F_{T_{0}} w_{T_{0}}\right\} \\
& \quad=(z-\bar{z})^{\top}\left\{\left[\mathcal{J}_{T_{c}}-\mathcal{R}_{T}+F_{T} \mathcal{T}_{T} F_{T}^{\top}\right](z-\bar{z})\right. \\
&\left.\quad+\mathcal{J}_{T_{u}}(u) z-\mathcal{J}_{T_{u}}(\bar{u}) \bar{z}\right\} \\
& \dot{\overline{\mathcal{H}}}_{T}(s)=-(z-\bar{z})^{\top} \mathcal{R}_{T}(z-\bar{z}) \\
& \quad+(z-\bar{z})^{\top}\left\{\mathcal{J}_{T_{u}}(u) z-\mathcal{J}_{T_{u}}(\bar{u}) \bar{z}\right\} .
\end{aligned}
$$

Where the definition (72) and the skew-symmetry of the matrix $\left[\mathcal{J}_{T_{c}}+F_{T} \mathcal{T}_{T} F_{T}^{\top}\right]$ are used to obtain the final equation. Therefore, for the second part of equation Eq. (74), we have:

$$
\begin{aligned}
& (z-\bar{z})^{\top}\left\{\mathcal{J}_{T_{u}}(u) z-\mathcal{J}_{T_{u}}(\bar{u}) \bar{z}\right\} \\
& =(z-\bar{z})^{\top}\left\{\mathcal{J}_{T_{u}}(u)(z-\bar{z})+\mathcal{J}_{T_{u}}(u) \bar{z}-\mathcal{J}_{T_{u}}(\bar{u}) \bar{z}\right\} \\
& =(z-\bar{z})^{\top}\left\{\mathcal{J}_{T_{u}}(u-\bar{u})\right\} \bar{z}
\end{aligned}
$$

Then, recalling the equations (47), we have

$$
\begin{aligned}
& (z-\bar{z})^{\top}\left\{\mathcal{J}_{T_{u}}(u-\bar{u})\right\} \bar{z} \\
& =(\lambda-\bar{\lambda})^{\top}\left\{\mathcal{J}_{S_{u}}(u-\bar{u})\right\} \bar{\lambda} \\
& =(\lambda-\bar{\lambda})^{\top}\left\{A_{S}(\bar{s})\right\}(u-\bar{u})=\tilde{y}_{S_{A}}^{\top} \tilde{u} .
\end{aligned}
$$

Where $\tilde{y}_{S_{A}}=A_{S}^{\top}(\bar{s})\left(\nabla \mathcal{H}_{S}(s)-\nabla \mathcal{H}_{S}(\bar{s})\right)$. Therefore, the overall port-Hamiltonian system Eq. (69) verifies the incremental passivity with shifted-Hamiltonian storage function $\overline{\mathcal{H}}_{T}$.

\section{Microgrid closed-loop stability analysis and control in grid-connected mode}

In this section, we show that both the microsource dynamic as well as the decentralized controller admit a port-Hamiltonian representation (Fig. 6), and in particular are passive systems. As a result, the interconnection between the controller and the nonlinear microsource system is power-preserving, implying passivity of the closed loop system as well.

Remark 7 In grid-connected mode, it is assumed that the frequency and voltage of microgrid are stabilized by main grid. Therefore, the microsources work to manage active and reactive injected into micro-grid.

In this section, in order to control the microsource output current $i_{S_{d q_{i}}}$ and $d c$ voltage the following decentralized 


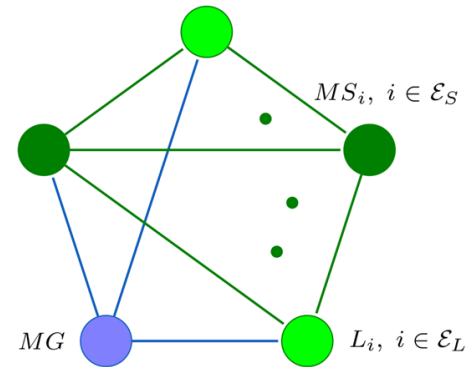

Fig. 6 Microgrid in grid-connected mode

passive controller (proportional-integral) is considered:

$$
\begin{aligned}
\dot{\eta}_{d q_{i}} & =u_{C_{i}}=-y_{B_{i}}, \\
u_{d q_{i}} & =y_{C_{i}}=-K_{P_{d q_{i}}} y_{B_{i}}+K_{I_{d q_{i}}} \eta_{d q_{i}}, \\
y_{B_{i}} & =B_{i}^{\top}\left(\bar{x}_{i}\right) \nabla \mathcal{H}_{S}\left(x_{i}\right) \\
& =\left[\bar{v}_{d c_{i}} \mathbb{1}_{2} \bar{v}_{d c_{i}} \bar{\omega} C_{i} \mathbf{J}-\bar{i}_{S_{d q_{i}}}\right] \nabla \mathcal{H}_{S}\left(x_{i}\right) .
\end{aligned}
$$

In compact form, we have:

$$
\begin{aligned}
\dot{\boldsymbol{\eta}}_{C, d q} & =u_{C}=-y_{B}, \\
u & =\operatorname{col}\left\{u_{d q_{i}}\right\}=y_{C}=-K_{P} y_{B}+K_{I} \eta_{C, d q}
\end{aligned}
$$

Where $K_{P}=b d g\left\{K_{P_{d q_{i}}}\right\}, K_{I}=b d g\left\{K_{I_{d q_{i}}}\right\}$ and $\mathcal{H}_{C_{i}}=$ $\frac{1}{2}\left(\eta_{C_{d q_{i}}}\right)^{\top} K_{I_{d q_{i}}}\left(\eta_{C_{d q_{i}}}\right)$.

In this section, the combination of controller dynamic (decentralized passive controller) (78) with other microgrid unit dynamics is analyzed in port-Hamiltonian form. Hence, the aggregated model of the microsource (12) and controller dynamics (78) can be then written as:

$$
\begin{aligned}
{\left[\begin{array}{c}
\dot{\boldsymbol{\eta}}_{S, d q} \\
\dot{\boldsymbol{\phi}}_{S, d q} \\
\dot{\boldsymbol{q}}_{S, d q} \\
\dot{\boldsymbol{q}}_{d c, S}
\end{array}\right]=} & \left(\mathcal{J}_{S C_{u}}+\mathcal{J}_{S C_{c}}-\mathcal{R}_{S C}\right)\left[\begin{array}{c}
\frac{\partial \mathcal{H}_{S C}}{\partial \boldsymbol{\eta}_{S, d q}} \\
\frac{\partial \mathcal{H}_{S C}}{\partial \boldsymbol{\phi}_{S, d q}} \\
\frac{\partial \mathcal{H} \mathcal{H}_{S C}}{\partial \boldsymbol{q}_{S, d q}} \\
\frac{\partial \mathcal{H} \mathcal{H}_{S C}}{\partial \boldsymbol{q}_{d c, S}}
\end{array}\right] \\
+ & F_{S C} \boldsymbol{i}_{S, d q}+E_{S C} \boldsymbol{r}_{S, d q} \\
& +F_{S C_{0}} \boldsymbol{i}_{S_{0}}, \boldsymbol{v}_{S, d q}=F_{S C}^{\top} \nabla \mathcal{H}_{S C}\left(x_{S C}\right)
\end{aligned}
$$

Where $u_{d q_{i}}=y_{C_{i}}=-K_{P_{d q_{i}}} y_{B_{i}}+K_{I_{d q_{i}}} \eta_{d q_{i}}$. In addition, the new Hamiltonian energy function is obtained by combination of microsource and controller energy functions, Hence, we have:

$$
\mathcal{H}_{S C}:=\mathcal{H}_{S}+\mathcal{H}_{C}
$$

Similar to Section 4.5, the overall model can be obtained by collecting the unit dynamics (3), (32), (45) and (79) and also interconnection overall law (68), so we have (Fig. 7):

$$
\begin{aligned}
0= & w_{G}-\bar{w}_{G}, \\
\dot{x}_{T}= & {\left[\mathcal{J}_{T_{u}}+\mathcal{J}_{T_{c}}-\mathcal{R}_{T}+F_{T} \mathcal{T}_{T} F_{T}^{\top}\right] \nabla \mathcal{H}_{T C}\left(x_{T}\right) } \\
& +G_{T} \bar{y}_{G}+E_{T} r_{T}+F_{T_{0}} w_{T_{0}}, \\
y_{T_{A}}= & A_{T}^{\top}\left(x_{T}\right) \nabla \mathcal{H}_{T C}\left(x_{T}\right), \\
y_{T}= & F_{T}^{\top} \nabla \mathcal{H}_{T C}\left(x_{T}\right) \\
y_{G}= & \bar{y}_{G}, \\
y_{T_{0}}= & F_{T_{0}}^{\top} \nabla \mathcal{H}_{T C}\left(x_{T}\right),
\end{aligned}
$$

Where the numbers are obtained by collecting the (14), (27) and (40):

$$
\begin{aligned}
& n_{T}:=n_{S C}+n_{L}+n_{D}, \quad m_{T}:=m_{S}, \\
& p_{T}:=p_{S}+p_{L}+p_{D}, \quad p_{T_{0}}:=p_{S_{0}} .
\end{aligned}
$$

Where $n_{S C}=n_{S}+n_{C}$ with $n_{C}=\sum_{i=1}^{s} p_{i}$.

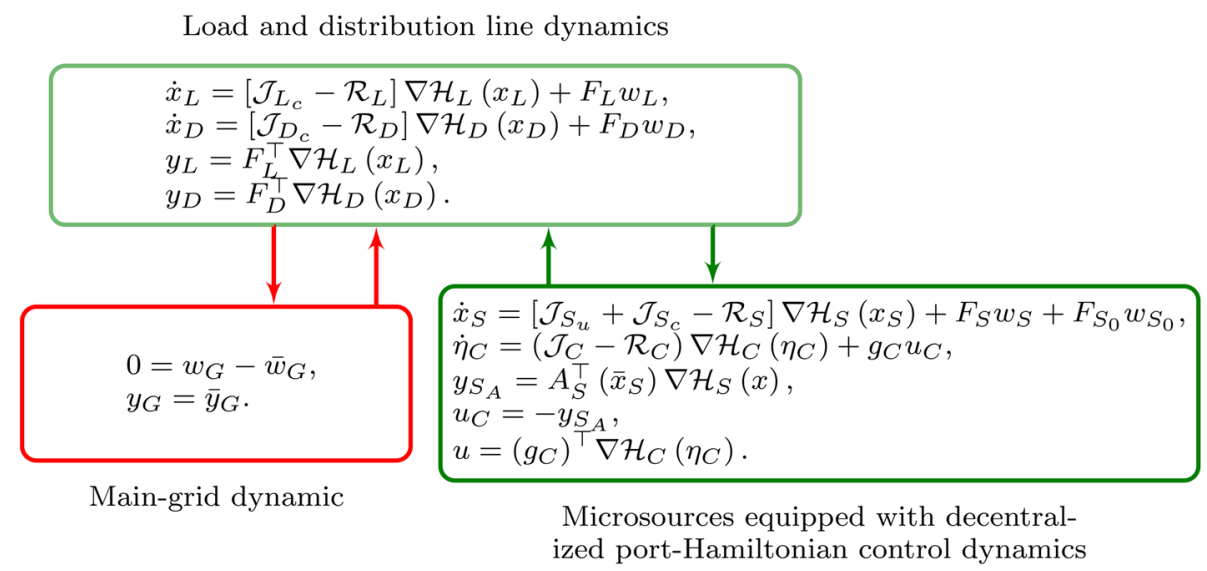

Fig. 7 Overall microgrid dynamic in grid-connected mode: microsources equipped with decentralized port-Hamiltonian control dynamics 
In addition, collecting interconnection matrices of the microgrid unit dynamics (29), (42) and (79):

$$
\begin{aligned}
& \mathcal{J}_{T_{u}}:=b d g\left\{\mathcal{J}_{S C_{u}}, 0,0\right\}, \mathcal{J}_{T_{c}}:=b d g\left\{\mathcal{J}_{S C_{c}}, \mathcal{J}_{L_{c}}, \mathcal{J}_{D_{c}}\right\} \text {, } \\
& \mathcal{J}_{S C_{u}}:=b d g\left\{\left[\begin{array}{cccc}
\mathbb{O}_{2} & -\bar{v}_{d c_{i}} \mathbb{D}_{2} & -\bar{v}_{d c_{i}} \bar{\omega} C_{i} \mathbf{J} \bar{i}_{S_{d q_{i}}} \\
v_{d c_{c}} \mathrm{D}_{2} & \mathbb{O}_{2} & \mathbb{O}_{2} & -\alpha \\
v_{d c_{i}} \bar{\omega} C_{i} \mathbf{J} & \mathbb{O}_{2} & \mathbb{O}_{2} & 0_{21} \\
-i_{S_{d q_{i}}}^{\top} & \alpha^{\top} & 0_{12} & 0
\end{array}\right]\right\} \text {, } \\
& \mathcal{J}_{S C_{c}}:=b d g\left\{\left[\begin{array}{cccc}
\mathbb{O}_{2} & \mathbb{0}_{2} & \mathbb{0}_{2} & 0_{21} \\
\mathbb{O}_{2} & \bar{\omega} L_{i} \mathbf{J} & -\mathbb{D}_{2} & 0_{21} \\
\mathbb{O}_{2} & \mathbb{D}_{2} & \bar{\omega} C_{i} \mathbf{J} & 0_{21} \\
0_{12} & 0_{12} & 0_{12} & 0
\end{array}\right]\right\} \text {, } \\
& \mathcal{J}_{L_{c}}:=b d g\left\{\bar{\omega} C_{i} \mathbf{J}\right\}, \mathcal{J}_{D_{c}}:=\operatorname{bdg}\left\{\bar{\omega} L_{i} \mathbf{J}\right\} .
\end{aligned}
$$

Where $\alpha=K_{P_{d q_{i}}} y_{B_{i}}$. Collecting dissipation matrices of the microgrid unit dynamics (29), (42) and (79):

$$
\begin{aligned}
& \mathcal{R}_{T}:=b d g\left\{\mathcal{R}_{S C}, \mathcal{R}_{L}, \mathcal{R}_{D}\right\}, \\
& \mathcal{R}_{S C}:=b d g\left\{\left[\begin{array}{cccc}
\mathbb{O}_{2} & \mathbb{O}_{2} & \mathbb{O}_{2} & 0_{21} \\
\mathbb{O}_{2} & R_{i} \mathbb{D}_{2} & \mathbb{O}_{2} & 0_{21} \\
\mathbb{O}_{2} & \mathbb{O}_{2} & \mathbb{O}_{2} & 0_{21} \\
0_{12} & 0_{12} & 0_{12} & G_{d c_{i}}
\end{array}\right]\right\} \text {, } \\
& \mathcal{R}_{L}:=b d g\left\{R_{i} \rrbracket_{2}\right\}, \quad \mathcal{R}_{D}:=b d g\left\{R_{i} \rrbracket_{2}\right\} .
\end{aligned}
$$

and collecting interaction port matrices of the microgrid unit dynamics (30), (43) and (79):

$$
\begin{array}{r}
F_{T}:=b d g\left\{F_{G}, F_{S C}, 0, F_{D}\right\}, \\
F_{G}:=1, \quad F_{S C}:=b d g\left\{\left[\begin{array}{c}
\mathbb{O}_{2} \\
\mathbb{O}_{2} \\
-\mathbb{D}_{2} \\
0_{12}
\end{array}\right]\right\}, \\
F_{L}:=b d g\left\{\square_{2}\right\}, F_{D}:=b d g\left\{\square_{2}\right\} .
\end{array}
$$

and collecting microsource port matrices (79):

$$
F_{T_{0}}:=b d g\left\{F_{S C_{0}}, 0,0\right\} ; F_{S C_{0}}:=b d g\left\{\left[\begin{array}{c}
0_{21} \\
0_{21} \\
0_{21} \\
1
\end{array}\right]\right\} \text {, }
$$

and collecting reference signal matrix (79):

$$
E_{T}^{\top}=b d g\left\{\left[\begin{array}{llll}
g_{C_{i}} \mathbb{Q}_{2} & \mathbb{O}_{2} & \mathbb{O}_{2} & 0_{12}
\end{array}\right]\right\} .
$$

Proposition 2 Suppose now that there exists an equilibrium $\bar{x}_{T}$, refer to system (81). Then, the following shifted
Hamiltonian energy function transfers the state variable $x_{T}$ to the desired equilibrium $\bar{x}_{T}$.

$$
\overline{\mathcal{H}}_{T C}(s):=\mathcal{H}_{T C}(s)-\left(\nabla \mathcal{H}_{T C}(\bar{s})\right)^{\top}(s-\bar{s})-\mathcal{H}_{T C}(\bar{s}) .
$$

Proof By defining $\tau=\nabla \mathcal{H}_{C}\left(\eta_{d q}\right), \bar{\tau}=\nabla \mathcal{H}_{C}\left(\bar{\eta}_{d q}\right)$ and recalling the shifted Hamiltonian energy function (73) and equations (75) and (81), we then have:

$$
\begin{aligned}
\dot{\overline{\mathcal{H}}}_{T C}(s) & =\left(\nabla \mathcal{H}_{T C}(s)-\nabla \mathcal{H}_{T C}(\bar{s})\right)^{\top} \dot{s} \\
& =(z-\bar{z})^{\top}\left\{\left[\mathcal{J}_{T_{u}}+\mathcal{J}_{T_{c}}-\mathcal{R}_{T}+F_{T} \mathcal{T}_{T} F_{T}^{\top}\right] z\right. \\
& \left.+G_{T} \bar{y}_{G}+F_{T_{0}} w_{T_{0}}\right\}+(\tau-\bar{\tau})^{\top} \dot{\eta}_{d q} \\
& =-(z-\bar{z})^{\top} \mathcal{R}_{T}(z-\bar{z}) \\
& +(z-\bar{z})^{\top}\left\{\mathcal{J}_{T_{u}}(u-\bar{u})\right\} \bar{z}-\left(\eta_{d q}-\bar{\eta}_{d q}\right)^{\top} K_{I_{d q}}^{\top} y_{B} \\
& =-(z-\bar{z})^{\top} \mathcal{R}_{T}(z-\bar{z})+(\lambda-\bar{\lambda})^{\top}\left\{B_{S}(\bar{s})\right\}(u-\bar{u}) \\
& -\left\{(u-\bar{u})^{\top}+(\lambda-\bar{\lambda})^{\top}\left\{B_{S}(\bar{s})\right\} K_{P_{d q}}\right\} B_{S}^{\top}(\bar{s})(\lambda-\bar{\lambda}) \\
& =-(z-\bar{z})^{\top} \mathcal{R}_{T}(z-\bar{z}) \\
& -(\lambda-\bar{\lambda})^{\top}\left\{B_{S}(\bar{s})\right\} K_{P_{d q}}\left\{B_{S}(\bar{s})\right\}^{\top}(\lambda-\bar{\lambda}) \\
& =-(z-\bar{z})^{\top} \mathcal{R}_{T}(z-\bar{z})-\tilde{y}_{S_{B}}^{\top} K_{P_{d q}} \tilde{y}_{S_{B}} .
\end{aligned}
$$

Therefore, the overall port-Hamiltonian system (81) with the decentralized passive (proportional-integral) control dynamic using microsource output current (78) satisfies the incremental passivity.

\section{Microgrid closed-loop stability analysis and control in Islanded mode}

In islanded mode, the voltage and frequency of microgrid are stabilized by the inverter-based microsources (Fig. 8). Therefore, in order to control the frequency and voltage as well as regulate active and reactive power (or current) the microsources are divided into grid-forming (frequency control) $i \in \mathcal{E}_{S_{F}}$ and grid feeding $i \in \mathcal{E}_{S_{V}}$ (voltage and power) parts. In first part, the $d c$ voltage of microsource

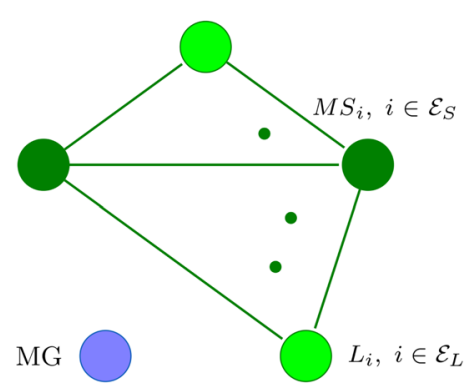

Fig. 8 Microgrid in islanding mode 
is used to capture the dynamic of microsource frequency control $i \in \mathcal{E}_{S_{F}}$ (see also [104]). Therefore, considering $\dot{\theta}_{i}=\omega_{i}=q_{d q_{i}}$ and recalling the $d c$-side dynamic of microsource (12):

$$
\dot{q}_{d c_{i}}=\left(-u_{d q_{i}}^{\top}\right) i_{d q_{i}}-G_{d c_{i}}\left(C_{d c_{i}}^{-1} q_{d c_{i}}\right)+i_{S_{0 i}} .
$$

Hence, the dynamic of (grid-forming) frequency control can be described as follows:

$$
\begin{aligned}
\dot{\theta}_{i} & =\omega_{i}, \\
\dot{\omega}_{i} & =-\left(G_{d c_{i}} C_{d c_{i}}^{-1}\right) \omega_{i}-\left(u_{d q_{i}}^{\top}\right) i_{d q_{i}}+i_{S_{0 i}} \\
& =-d \omega_{i}-u_{C_{i}}+\bar{u}_{C_{i}} .
\end{aligned}
$$

Where $u_{C_{i}}=\left(u_{d q_{i}}^{\top}\right) i_{d q_{i}}$ and $i_{S_{0 i}}=\bar{u}_{C_{i}}$. The Hamiltonian energy function of the (grid-forming) microsource $\mathcal{H}\left(\phi_{d q_{i}}, q_{d q_{i}}, \omega_{i}\right)$, which denotes the total energy, is given by:

$$
\mathcal{H}\left(x_{i}\right)=\frac{1}{2}\left(\phi_{d q_{i}}^{\top}\left(L \rrbracket_{2}\right)^{-1} \phi_{d q_{i}}+q_{d q_{i}}^{\top}\left(C \rrbracket_{2}\right)^{-1} q_{d q_{i}}+\omega_{i}^{2}\right) .
$$

where $\phi_{d q_{i}}$ is the flux linkage across the inductors, $q_{d q_{i}}$ is the charge in the capacitors and $\omega_{i}$ is angle frequency.

Therefore, the aggregated model of the microsource dynamic (12) $i \in \mathcal{E}_{S_{F}}$ and the frequency controller dynamic (91) can be then written as:

$$
\begin{aligned}
{\left[\begin{array}{c}
\dot{\boldsymbol{\theta}}_{S} \\
\dot{\boldsymbol{\omega}}_{S} \\
\dot{\boldsymbol{\phi}}_{S, d q} \\
\dot{\boldsymbol{q}}_{S, d q}
\end{array}\right] } & =\left(\mathcal{J}_{S F C_{u}}+\mathcal{J}_{S F C_{c}}-\mathcal{R}_{S C}\right)\left[\begin{array}{c}
\frac{\partial \mathcal{H}_{S F C}}{\partial \boldsymbol{\theta}_{S, d q}} \\
\frac{\partial \mathcal{H}_{S F C}}{\partial \boldsymbol{\omega}_{S, d q}} \\
\frac{\partial \mathcal{H}_{S F C}}{\partial \boldsymbol{\phi}_{S, d q}} \\
\frac{\partial \mathcal{H}_{S F C}}{\partial \boldsymbol{q}_{S, d q}}
\end{array}\right] \\
& +F_{S F C} \boldsymbol{i}_{S, d q}
\end{aligned}
$$

Where we have:

$$
\begin{aligned}
& \mathcal{J}_{S F C_{u}}=b d g\left\{\left[\begin{array}{cccc}
0 & 1 & 0_{12} & 0_{12} \\
1 & 0 & \hat{m}^{\top}\left(\theta_{i}\right) \tilde{y}_{z_{i}} & 0_{12} \\
0_{21}-\left[\hat{m}\left(\theta_{i}\right)\right] \tilde{y}_{z_{i}} & \mathbb{O}_{2} & 0_{2} \\
0 & 0_{21} & \mathbb{0}_{2} & 0_{2}
\end{array}\right]\right\}, \\
& \mathcal{J}_{S F C_{c}}=b d g\left\{\left[\begin{array}{cccc}
0 & 0 & 0_{2} & 0^{21} \\
0 & 0 & 0_{12} & 0_{21} \\
0_{21} & 0_{21} & \bar{\omega} L_{i} \mathbf{J} & 0_{21} \\
0_{21} & 0_{21} & 0_{2} & \bar{\omega} C_{i} \mathbf{J}
\end{array}\right]\right\}, \\
& \mathcal{R}_{S F C}=b d g\left\{\left[\begin{array}{cccc}
0 & 0 & 0_{12} & 0_{12} \\
0 & d_{i} & 0_{12} & 0_{12} \\
0_{21} & 0_{21} & R_{i} \mathbb{D}_{2} & 0_{2} \\
0_{21} & 0_{21} & 0_{2} & 0_{2}
\end{array}\right]\right\}, \\
& F_{S F C}=b d g\left\{\left[\begin{array}{c}
0_{2} \\
\mathbb{O}_{2} \\
-\mathbb{D}_{2} \\
0_{12}
\end{array}\right]\right\} .
\end{aligned}
$$

In addition, the passive auxiliary output is defined as follows:

$$
\begin{aligned}
y_{z_{i}} & =Z^{\top}(\bar{s}) \nabla \mathcal{H}_{S F C}(s)=\bar{i}_{d q_{i}} \omega_{i}-\left(\bar{\omega}_{i} \rrbracket_{2}\right) i_{d q_{i}} \\
& =\left[\begin{array}{lll}
0_{21} & \bar{i}_{d q_{i}}-\bar{\omega}_{i} \rrbracket_{2} & \mathbb{D}_{2}
\end{array}\right] \nabla \mathcal{H}_{S F C}(s) .
\end{aligned}
$$

In second part $i \in \mathcal{E}_{S_{V}}$, as mentioned in Section 6, in order to control (grid-feeding) microsource output current and voltage $\left(i_{S_{d q_{i}}}, v_{S_{d q_{i}}}\right)$ and $d c$ voltage the following decentralized passive controller (proportional-integral) is considered:

$$
\begin{aligned}
\dot{\eta}_{d q_{i}} & =u_{C_{i}}=-y_{M_{i}}, \\
u_{d q_{i}} & =y_{C_{i}}=-K_{P_{d q_{i}}} y_{M_{i}}+K_{I_{d q_{i}}} \eta_{d q_{i}}, \\
y_{M_{i}} & =M_{i}^{\top}\left(\bar{x}_{i}\right) \nabla \mathcal{H}_{S V}\left(\phi_{d q_{i}}, q_{d q_{i}}, q_{d c_{i}}\right) \\
& =\left[\bar{v}_{d c_{i}} \square_{2} \bar{v}_{d c_{i}}\left(\omega C_{i} \mathbf{J}+\square_{2}\right)-\bar{i}_{S_{d q_{i}}}\right] \nabla \mathcal{H}_{S V}\left(x_{i}\right)
\end{aligned}
$$

In compact form, we have:

$$
\begin{aligned}
\dot{\boldsymbol{\eta}}_{C, d q} & =u_{C}=-y_{M}, \\
u & =\operatorname{col}\left\{u_{d q_{i}}\right\}=y_{C}=-K_{P} y_{M}+K_{I} \boldsymbol{\eta}_{C, d q} .
\end{aligned}
$$

By defining $K_{P}=b d g\left\{K_{P_{d q_{i}}}\right\}, K_{I}=b d g\left\{K_{I_{d q_{i}}}\right\}$ and $\mathcal{H}_{C_{i}}=\frac{1}{2}\left(\eta_{C_{d q_{i}}}\right)^{\top} K_{I_{d q_{i}}}\left(\eta_{C_{d q_{i}}}\right)$, The new Hamiltonian energy function is obtained by combination of microsource and controller energy functions, Hence, we have:

$$
\mathcal{H}_{S V C}:=\mathcal{H}_{S V}+\mathcal{H}_{C}
$$

Therefore, the aggregated model of the microsource (12) $i \in \mathcal{E}_{S_{V}}$ and controller dynamic (96) can be then written as:

$$
\begin{aligned}
{\left[\begin{array}{c}
\dot{\boldsymbol{\eta}}_{S, d q} \\
\dot{\boldsymbol{\phi}}_{S, d q} \\
\dot{\boldsymbol{q}}_{S, d q} \\
\dot{\boldsymbol{q}}_{d c, S}
\end{array}\right] } & =\left(\mathcal{J}_{S V C_{u}}+\mathcal{J}_{S V C_{c}}-\mathcal{R}_{S V C}\right)\left[\begin{array}{c}
\frac{\partial \mathcal{H}_{S V C}}{\partial \boldsymbol{\eta}_{S, d q}} \\
\frac{\partial \mathcal{H}_{S V C}}{\partial \boldsymbol{\phi}_{S, d q}} \\
\frac{\partial \mathcal{H}_{S V C}}{\partial \boldsymbol{q}_{S, d q}} \\
\frac{\partial \mathcal{H}_{S V C}}{\partial \boldsymbol{q}_{d c, S}}
\end{array}\right] \\
& +F_{S V C} \boldsymbol{i}_{S, d q}+E_{S V C} \boldsymbol{r}_{S, d q}+F_{S V C_{0}} \boldsymbol{i}_{S_{0}}, \\
\boldsymbol{v}_{S, d q} & =F_{S C}^{\top} \nabla \mathcal{H}_{S V C}\left(x_{S C}\right)
\end{aligned}
$$




$$
\begin{aligned}
& \mathcal{J}_{S V C_{u}}=b d g\left\{\left[\begin{array}{cccc}
\mathbb{O}_{2} & -\bar{v}_{d c_{i}} \mathbb{D}_{2} & -\beta & \bar{i}_{S_{d q_{i}}} \\
v_{d c_{i}} \mathbb{D}_{2} & \mathbb{O}_{2} & \mathbb{O}_{2} & -K_{P_{d q_{i}}} y_{M_{i}} \\
\beta^{\top} & \mathbb{O}_{2} & \mathbb{O}_{2} & 0_{12} \\
-i_{S_{d q_{i}}}^{\top} & y_{M_{i}}^{\top} K_{P_{d q_{i}}}^{\top} & 0_{12} & 0
\end{array}\right]\right\} \\
& \mathcal{J}_{S V C_{c}}=b d g\left\{\left[\begin{array}{cccc}
\mathbb{O}_{2} & \mathbb{O}_{2} & \mathbb{O}_{2} & 0_{21} \\
\mathbb{O}_{2} & \omega L_{i} \mathbf{J} & -\mathbb{D}_{2} & 0_{21} \\
\mathbb{O}_{2} & \mathbb{O}_{2} & \omega C_{i} \mathbf{J} & 0_{21} \\
0_{12} & 0_{12} & 0_{12} & 0
\end{array}\right]\right\} \\
& \mathcal{R}_{S V C}=b d g\left\{\left[\begin{array}{cccc}
\mathbb{O}_{2} & \mathbb{O}_{2} & \mathbb{O}_{2} & 0_{21} \\
\mathbb{O}_{2} & \mathbb{R}_{i} \mathbb{D}_{2} & \mathbb{O}_{2} & 0_{21} \\
\mathbb{O}_{2} & \mathbb{O}_{2} & \mathbb{O}_{2} & 0_{21} \\
0_{12} & 0_{12} & 0_{12} & G_{d c_{i}}
\end{array}\right]\right\} \\
& F_{S V C}=b d g\left\{\left[\begin{array}{c}
\mathbb{O}_{2} \\
\mathbb{O}_{2} \\
-\mathbb{D}_{2} \\
0_{12}
\end{array}\right]\right\}, E_{S V C}=b d g\left\{\left[\begin{array}{c}
\mathbb{0}_{2} \\
\mathbb{O}_{2} \\
\mathbb{O}_{2} \\
0_{12}
\end{array}\right]\right\} \\
& F_{S V C_{0}}=b d g\left\{\left[\begin{array}{c}
0_{21} \\
0_{21} \\
0_{21} \\
1
\end{array}\right]\right\} .
\end{aligned}
$$

Where $u_{d q_{i}}=y_{C_{i}}=-K_{P_{d q_{i}}} y_{M_{i}}+K_{I_{d q_{i}}} \eta_{d q_{i}}$ and $\beta=$ $\bar{v}_{d c_{i}}\left(\omega C_{i} \mathbf{J}+\mathbb{1}_{2}\right)$.

Similar to Section 4.5, the overall model can be obtained by collecting the microgrid unit dynamics (3), (32), (45), (93) and (99) and also the interconnection overall law (68), so we have (Fig. 9):

$$
\begin{aligned}
\dot{x}_{T} & =\left[\mathcal{J}_{T_{u}}+\mathcal{J}_{T_{c}}-\mathcal{R}_{T}+F_{T} \mathcal{T}_{T} F_{T}^{\top}\right] \nabla \mathcal{H}_{T C}\left(x_{T}\right) \\
& +E_{T} r_{T}+F_{T_{0}} w_{T_{0}}, \\
y_{T_{A}} & =A_{T}^{\top}\left(x_{T}\right) \nabla \mathcal{H}_{T C}\left(x_{T}\right), \\
y_{T} & =F_{T}^{\top} \nabla \mathcal{H}_{T C}\left(x_{T}\right), \\
y_{T_{0}} & =F_{T_{0}}^{\top} \nabla \mathcal{H}_{T C}\left(x_{T}\right) .
\end{aligned}
$$

Proposition 3 Suppose now that there exists an equilibrium $\bar{x}_{T}$, refer to system (101). Then, the following shifted Hamiltonian energy function transfers the state variable $x_{T}$ to the desired equilibrium $\bar{x}_{T}$.

$$
\overline{\mathcal{H}}_{T C}(s):=\mathcal{H}_{T C}(s)-\left(\nabla \mathcal{H}_{T C}(\bar{s})\right)^{\top}(s-\bar{s})-\mathcal{H}_{T C}(\bar{s}) .
$$

Proof By recalling the shifted Hamiltonian energy function (73) and equations (75) and (101), Hence, we have:

$$
\begin{aligned}
\overline{\mathcal{H}}_{T}(s) & :=\mathcal{H}_{T C}(s)-\left(\nabla \mathcal{H}_{T C}(\bar{s})\right)^{\top}(s-\bar{s})-\mathcal{H}_{T C}(\bar{s}), \\
\dot{\overline{\mathcal{H}}}_{T}(s) & =-(z-\bar{z})^{\top} \mathcal{R}_{T}(z-\bar{z}) \\
& +(z-\bar{z})^{\top}\left\{\mathcal{J}_{T_{u}}(s-\bar{s})\right\} z
\end{aligned}
$$

Hence, for first part of above equation, we also have:

$$
\begin{aligned}
& (z-\bar{z})^{\top}\left\{\mathcal{J}_{T_{u}}(s-\bar{s})\right\} z \\
& =(z-\bar{z})^{\top}\left\{\mathcal{J}_{T_{u}}(s-\bar{s})\right\}(z-\bar{z}) \\
& +(z-\bar{z})^{\top}\left\{\mathcal{J}_{T_{u}}(s-\bar{s})\right\} \bar{z}
\end{aligned}
$$

Therefore, by defining $\lambda=\nabla \mathcal{H}_{S C}(s)$ and $\bar{\lambda}=\nabla \mathcal{H}_{S C}(\bar{s})$ for second part of Eq. (103), we have:

$$
\begin{aligned}
& (z-\bar{z})^{\top}\left\{\mathcal{J}_{T_{u}}(s-\bar{s})\right\}(z-\bar{z}) \\
& =(\lambda-\bar{\lambda})^{\top}\left\{\mathcal{J}_{S_{u}}(s-\bar{s})\right\}(\lambda-\bar{\lambda})=0
\end{aligned}
$$

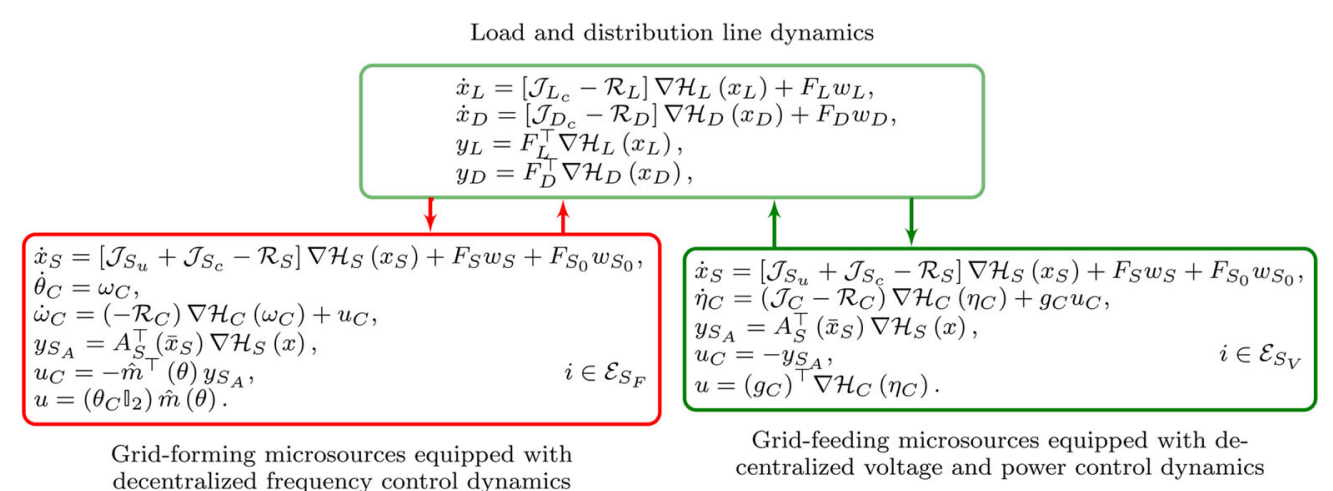

Fig. 9 Overall microgrid dynamic in islanding mode: grid-forming microsources equipped with decentralized frequency control dynamics and grid-feeding microsources equipped with decentralized voltage and power control dynamics 


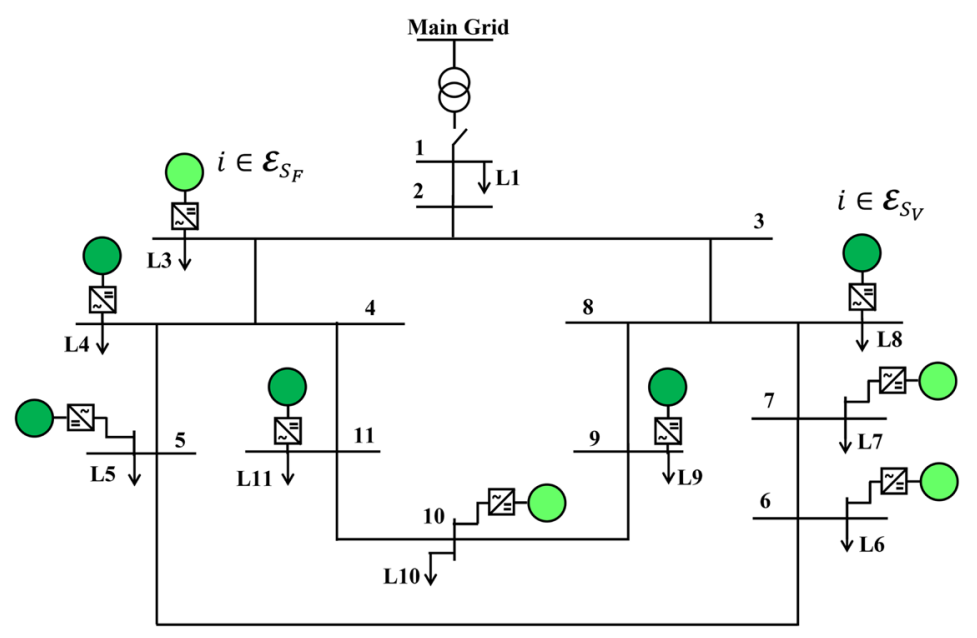

Fig. 10 Test system topology

Hence, by recalling Eq. (104), we have:

$$
\begin{aligned}
& (z-\bar{z})^{\top}\left\{\mathcal{J}_{T_{u}}(s-\bar{s})\right\} z \\
& =(\lambda-\bar{\lambda})^{\top}\left\{\mathcal{J}_{S_{u}}(s-\bar{s})\right\} \bar{\lambda} \\
& =-\sum_{i=1}^{s_{F}}\left\{\tilde{y}_{Z_{i}}^{\top}\left(M\left(\theta_{i}\right)\right) \tilde{y}_{Z_{i}}\right\}-\tilde{y}_{S_{B}}^{\top} K_{P_{d q}} \tilde{y}_{S_{B}} \\
& =0-\tilde{y}_{S_{B}}^{\top} K_{P_{d q}} \tilde{y}_{S_{B}}=-\tilde{y}_{S_{B}}^{\top} K_{P_{d q}} \tilde{y}_{S_{B}}
\end{aligned}
$$

Where $\tilde{y}_{S_{B}}=B_{S}^{\top}(\bar{s})\left(\nabla \mathcal{H}_{S V C}(s)-\nabla \mathcal{H}_{S V C}(\bar{s})\right)$ and $M\left(\theta_{i}\right)$ is defined as follows:

$$
M\left(\theta_{i}\right)=\left[\begin{array}{cccc}
0 & 0 & 0_{12} & 0_{12} \\
0 & 0 & \hat{m}^{\top}\left(\widetilde{\theta}_{i}\right) & 0_{12} \\
0_{21} & -\left[\hat{m}\left(\theta_{i}\right)\right] & \mathbb{O}_{2} & \mathbb{O}_{2} \\
0_{21} & 0_{21} & \mathbb{O}_{2} & \mathbb{O}_{2}
\end{array}\right]
$$

Therefore, the overall port-Hamiltonian system (101) with integrating the frequency control dynamic (91) and the voltage (power) control dynamic (94) satisfies the incremental passivity.

\section{Simulation}

In this section, the performance of the proposed control methodology is verified by a set of simulation studies. Therefore, a microgrid consisting of the ninemicrosources is chosen as a test system to demonstrate the effectiveness of the proposed control dynamics (see Fig. 10). The analysis is carried out based on the threephase Subnetwork 1 of the CIGRE benchmark medium voltage distribution network (see [105]). It should be noted, to satisfy the load demand in islanded mode, the power ratings of the generation units are modified. As illustrated in Fig. 10, this test microgrid has a meshed topology and consists of 11 main buses. Moreover, it contains a total of nine controllable microsources. In addition, the base apparent power rating $\left(S_{\text {base }}\right)$ is 4.3MVA.

We consider the following three scenarios.

\subsection{Grid-connected scenario}

As mention in Section 6, all microscources work as gridfeeding units. In addition, the microgrid contains a total of nine controllable microsources at buses 3, 4, 5, 6, 7, 8, 9, 10 and 11 (see Fig. 10). We associate to each microsource its apparent power rating $\bar{S}_{i}$. The $\bar{S}_{i}$ is given for each source in Table 1 . The active and reactive power setpoints $\left(\bar{P}_{i}\right.$ and $\bar{Q}_{i}$ ) relative to apparent power rating $\bar{S}_{i}$ are also mentioned in this table. Moreover, the load parameters are illustrated in Table 2.

Table 1 Grid-connected scenario: Microgrid parameters

\begin{tabular}{lllll}
\hline Bus No. & $\bar{S}_{i}[\mathrm{pu}]$ & $\bar{P}_{i} / \bar{S}_{i}[\mathrm{pu} / \mathrm{pu}]$ & $\bar{Q}_{i} / \bar{S}_{i}[\mathrm{pu} / \mathrm{pu}]$ & $\bar{V}_{d c}[\mathrm{v}]$ \\
\hline Bus 3 & 0.128 & 0.988 & 0.012 & 376.991 \\
Bus 4 & 0.070 & 1.000 & 0.000 & 500.000 \\
Bus 5 & 0.105 & 0.890 & 0.011 & 500.000 \\
Bus 6 & 0.116 & 0.792 & 0.198 & 376.991 \\
Bus 7 & 0.128 & 0.720 & 0.270 & 377.991 \\
Bus 8 & 0.116 & 0.800 & 0.020 & 500.000 \\
Bus 9 & 0.105 & 1.000 & 0.000 & 500.000 \\
Bus 10 & 0.128 & 0.720 & 0.270 & 376.991 \\
Bus 11 & 0.105 & 0.890 & 0.111 & 500.000 \\
\hline
\end{tabular}


Table 2 Load parameters

\begin{tabular}{lll}
\hline Bus No. & Active power [pu] & Reactive power [pu] \\
\hline Bus 1 & 0.0698 & 0.0010 \\
Bus 2 & 0.0000 & 0.0000 \\
Bus 3 & 0.0257 & 0.0019 \\
Bus 4 & 0.0954 & 0.0016 \\
Bus 5 & 0.1885 & 0.0035 \\
Bus 6 & 0.1303 & 0.0012 \\
Bus 7 & 0.1164 & 0.0019 \\
Bus 8 & 0.0722 & 0.0019 \\
Bus 9 & 0.0607 & 0.0021 \\
Bus 10 & 0.0745 & 0.0017 \\
Bus 11 & 0.0954 & 0.0021 \\
\hline
\end{tabular}

In addition, the decentralized power sharing (and voltage) controllers are equipped with the PI controllers (77). In this scenario, the controller parameters of the PI controllers are chosen as follows: for microsource output current $\left(i_{S_{d q_{i}}}\right)$ and output voltage control, $K_{P}=0.9 \rrbracket_{2}$ and
$K_{I}=20 \rrbracket_{2}$ as well as for frequency control, $K_{P}=10$ and $K_{I}=500$.

The simulation results are shown in Fig. 11. More precisely, the microgrid dynamic responses including frequency synchronization (the relative frequencies $\Delta f_{i}=$ $\left.\left(\omega_{i}-\bar{\omega}_{i}\right) /(2 \pi)\right)$, the power sharing (active/reactive power outputs relative to apparent power rating $P_{i} / S_{i}$ and $Q_{i} / S_{i}$ ) as well as $d c$ and $a c$ voltage tracking (amplitudes at microsource terminals) are illustrated.

The simulation results show that all trajectories converge to desired states demonstrating the stability analysis in Section 6. After a transient, the frequencies synchronize and the amplitudes of bus voltages become constant. The frequencies at the all microsource buses are converged to nominal value. The voltage amplitudes remain within $1 \pm 0.1 p u$ in steady-state. Therefore, the results demonstrate that the proposed controllers can regulate the microsource voltages with good tracking performance. The initial conditions have been chosen arbitrarily. Hence, the simulation result shows the stability of the decentralized frequency and voltage control dynamics, as given in (77). Furthermore, the simulation confirms that the
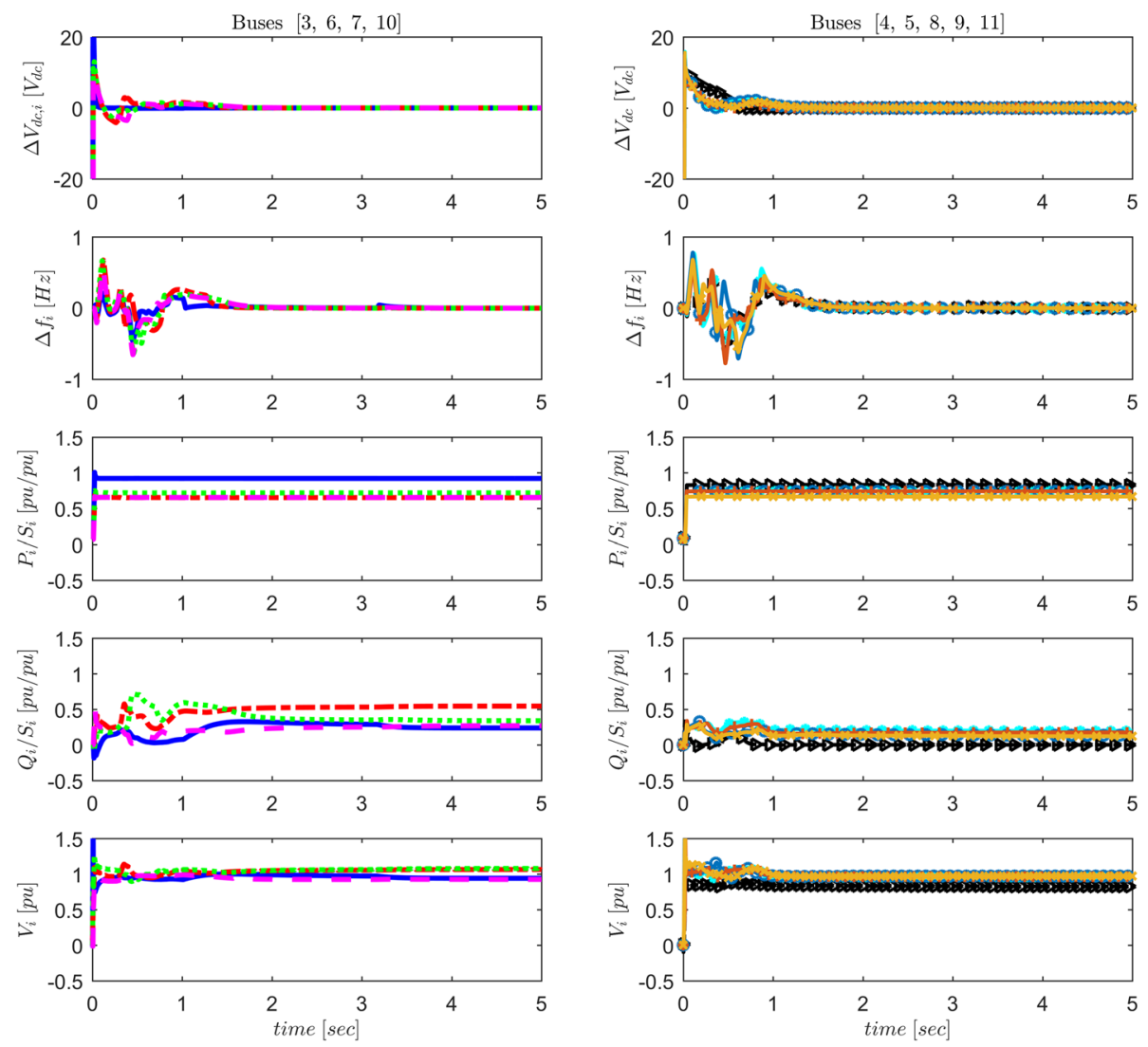

Fig. 11 Grid-connected scenario. The microsource dynamic responses including $d c$ voltage tracking, frequency synchronization, the power sharing as well as ac voltage tracking (amplitudes at output terminals). The lines correspond to the grid-feeding microsources at: bus 3, ' - ', bus $6,{ }^{\prime}--^{\prime}$, bus 7 ,

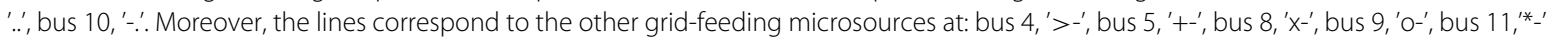


Table 3 Islanded scenario: Microgrid parameters

\begin{tabular}{|c|c|c|c|c|}
\hline Bus No. & $\bar{S}_{i}[\mathrm{pu}]$ & $\bar{P}_{i} / \bar{S}_{i}[\mathrm{pu} / \mathrm{pu}]$ & $\bar{Q}_{i} / \bar{S}_{i}[\mathrm{pu} / \mathrm{pu}]$ & $\bar{V}_{d c}[\mathrm{v}]$ \\
\hline Bus $3, i \in \mathcal{E}_{S_{F}}$ & 0.128 & 0.988 & 0.012 & 376.991 \\
\hline Bus $4, i \in \mathcal{E}_{S_{V}}$ & 0.070 & 1.000 & 0.000 & 500.000 \\
\hline Bus $5, i \in \mathcal{E}_{S_{V}}$ & 0.105 & 0.890 & 0.011 & 500.000 \\
\hline Bus $6, i \in \mathcal{E}_{S_{F}}$ & 0.116 & 0.792 & 0.198 & 376.991 \\
\hline Bus $7, i \in \mathcal{E}_{S_{F}}$ & 0.128 & 0.720 & 0.270 & 377.991 \\
\hline Bus $8, i \in \mathcal{E}_{S_{V}}$ & 0.116 & 0.800 & 0.020 & 500.000 \\
\hline Bus $9, i \in \mathcal{E}_{S_{V}}$ & 0.105 & 1.000 & 0.000 & 500.000 \\
\hline Bus $10, i \in \mathcal{E}_{S_{F}}$ & 0.128 & 0.720 & 0.270 & 376.991 \\
\hline Bus $11, i \in \mathcal{E}_{S_{V}}$ & 0.105 & 0.890 & 0.111 & 500.000 \\
\hline
\end{tabular}

decentralized control dynamics are suited to achieve the desired objective of frequency synchronization, voltage control and power sharing.

\subsection{Islaned scenario}

As illustrated in Fig. 10, the microgrid contains a total of nine controllable microsources four of which are gridforming microsources $\left(i \in \mathcal{E}_{S_{F}}\right.$ ) equipped with decentralized frequency and voltage controllers at buses $3,6,7$ and 10 and five of which are grid-feeding microsources $\left(i \in \mathcal{E}_{S_{V}}\right.$ ) equipped with decentralized power and voltage controllers at buses 4, 5, 8, 9 and 11. In addition, the microsource and control setpoints, $i \in \mathcal{E}_{S_{F}}$ and $i \in \mathcal{E}_{S_{V}}$, are given in Table 3. Moreover, the load parameters are illustrated in Table 2.

As mention in Section 7, for the grid-forming microsources $\left(i \in \mathcal{E}_{S_{F}}\right.$ ), the decentralized frequency (and voltage as well as power sharing) controllers are equipped with the PI controllers (91). In this scenario, the controller parameters of the PI controllers are chosen as follows: for microsource output current $\left(i_{S_{d q_{i}}}\right)$ and output voltage control, $K_{P}=0.9 \rrbracket_{2}$ and $K_{I}=$ $20 \rrbracket_{2}$ as well as for frequency control, $K_{P}=10$ and $K_{I}=500$.

In addition, for the grid-forming microsources $\left(i \in \mathcal{E}_{S_{V}}\right)$, the decentralized power sharing (and voltage) controllers are equipped with the PI controllers (95). In this scenario, the controller parameters of the PI controllers are chosen as follows: for microsource output current $\left(i_{S_{d q_{i}}}\right)$ and output voltage control, $K_{P}=0.9 \rrbracket_{2}$ and $K_{I}=20 \rrbracket_{2}$ as well as for frequency control, $K_{P}=10$ and $K_{I}=500$.
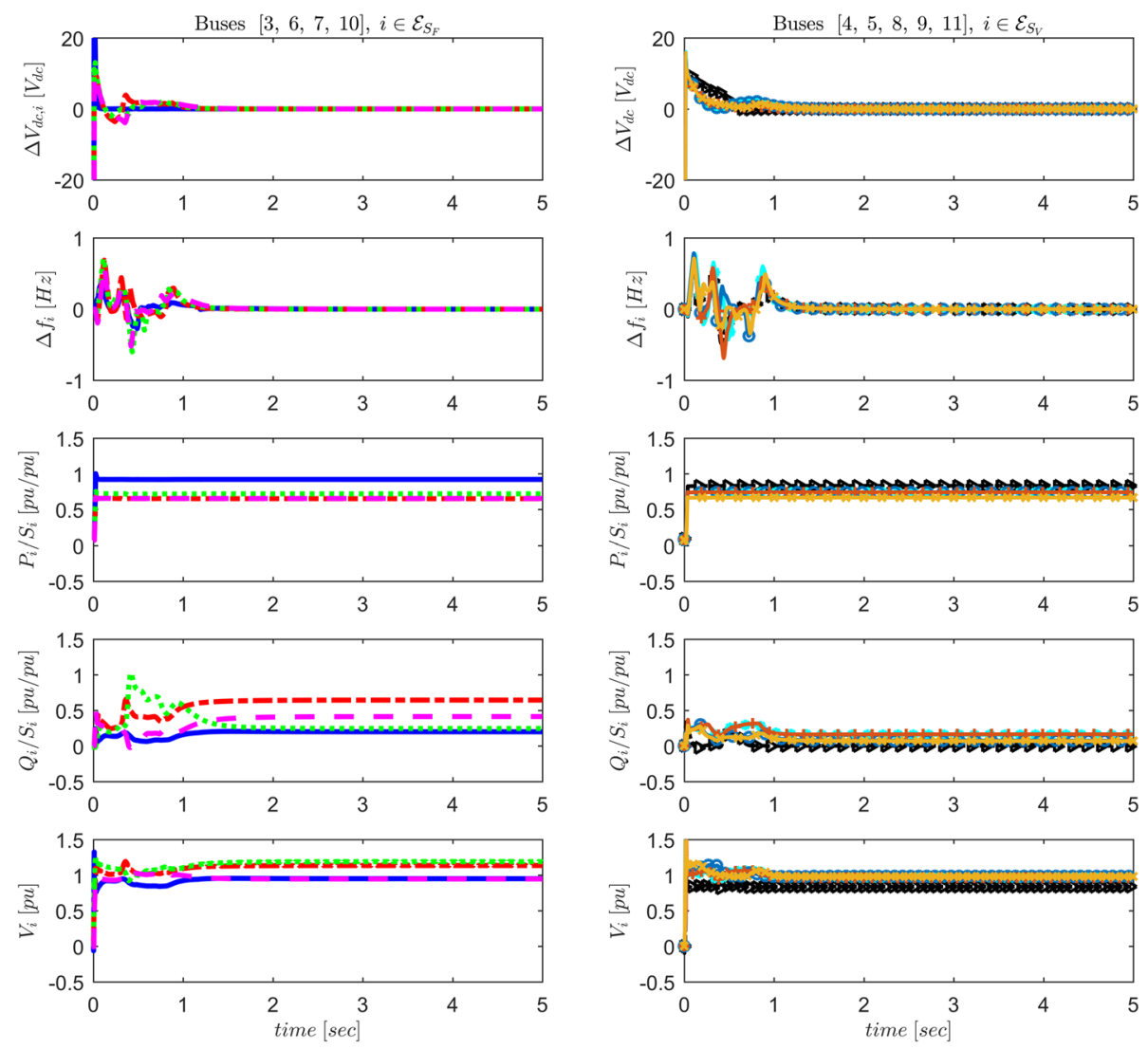

Fig. 12 Islanded scenario. The microsource dynamic responses including $d c$ voltage tracking, frequency synchronization, the power sharing as well as ac voltage tracking (amplitudes at output terminals). The lines correspond to the grid-forming microsources $i \in \mathcal{E}_{S_{F}}:$ bus $3,{ }^{\prime}-{ }^{\prime}$, bus $6,{ }^{\prime}--^{\prime}$, bus $7,{ }^{\prime} . .$, ,

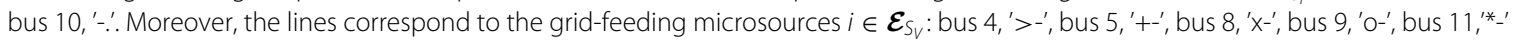


The simulation results are shown in Fig. 12. More precisely, the microgrid dynamic responses including frequency synchronization (the relative frequencies $\Delta f_{i}=$ $\left(\omega_{i}-\bar{\omega}_{i}\right) /(2 \pi)$, the power sharing (active/reactive power outputs relative to apparent power rating $P_{i} / S_{i}$ and $\left.Q_{i} / S_{i}\right)$ as well as $d c$ and $a c$ voltage tracking (amplitudes at microsource terminals) are illustrated, for $i \in \mathcal{E}_{S_{F}}$ and $i \in \mathcal{E}_{S_{V}}$

The simulation results show that all trajectories converge to desired states demonstrating the stability analysis in Section 7. After a transient, the frequencies synchronize and the amplitudes of bus voltages become constant. The frequencies at the all microsource buses $\left(i \in \mathcal{E}_{S_{F}}\right.$ and $i \in \mathcal{E}_{S_{V}}$ ) are converged to nominal value. The voltage amplitudes remain within $1 \pm 0.1 p u$ in steady-state. Therefore, the results demonstrate that the proposed controllers can regulate the microsource voltages with good tracking performance. The initial conditions have been chosen arbitrarily. Hence, the simulation result shows the stability of the decentralized frequency and voltage control dynamics, as given in (91) and (95). Furthermore, the simulation confirms that the decentralized control dynamics are suited to achieve the desired objective of frequency synchronization, voltage control and power sharing.

\subsection{Transition scenario}

The transient dynamics between grid-connected and islanded modes and vice versa are shown in Figs. 13 and 14. The switching is occurred at time $2.5 \mathrm{sec}$ via common coupling (CCP) switch. The controller and microsource parameters are selected similar to the gridconnected and islanded scenarios at previous subsections. After switching, all trajectories seamlessly converge to the desired states (see Figs. 13 and 14). In addition, the frequencies synchronize and the amplitudes of the all bus voltages become constant. The frequencies at the all microsource buses $\left(i \in \mathcal{E}_{S_{F}}\right.$ and $\left.i \in \mathcal{E}_{S_{V}}\right)$ are converged to nominal value. The voltage amplitudes remain within $1 \pm 0.1 p u$ in steady-state. Therefore, the results demonstrate that the proposed controllers can regulate the microsource voltages with good tracking performance.
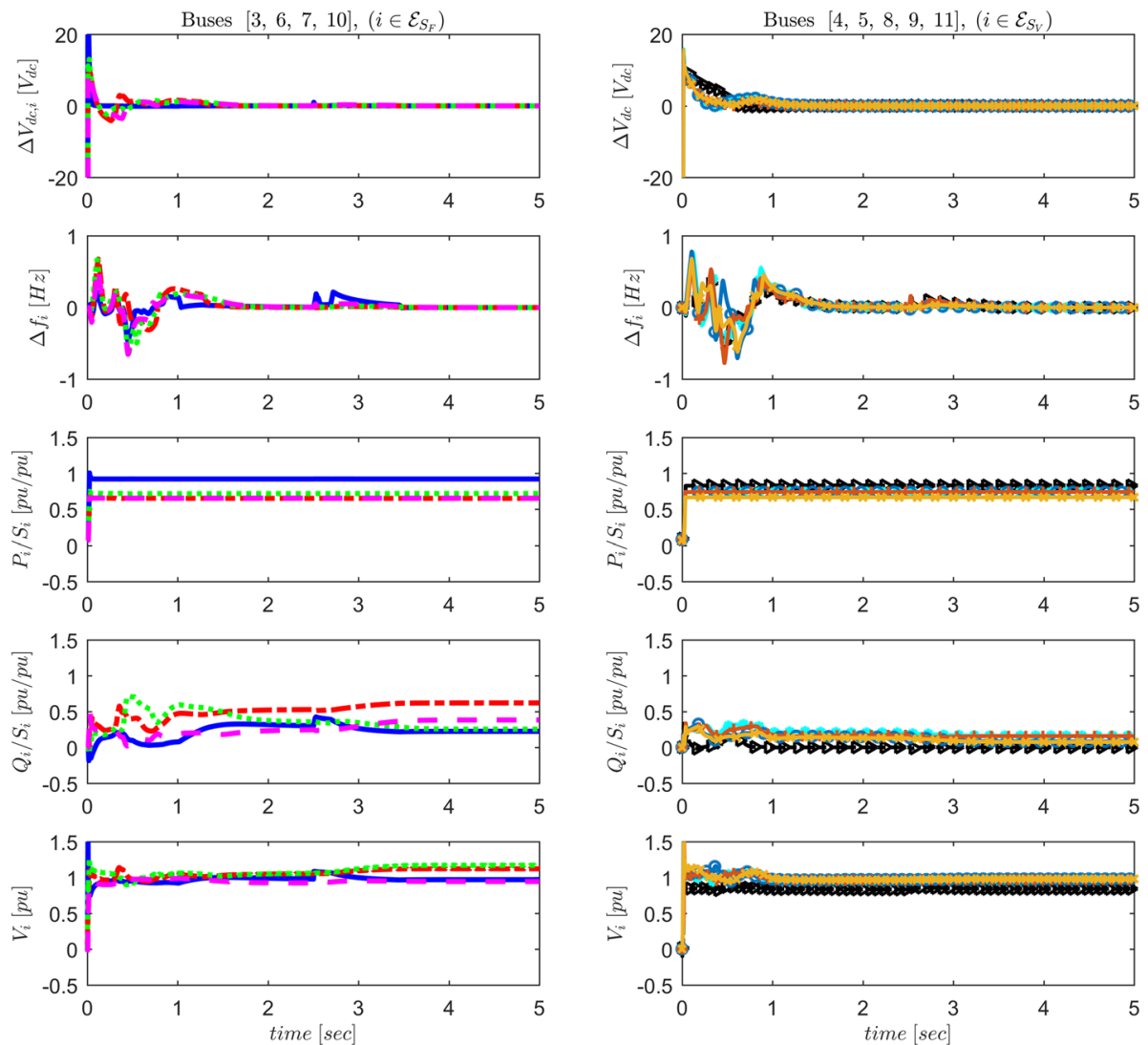

Fig. 13 Transition between grid-connected and islanded modes. The switching between grid-connected and islanded modes is occurred at time $2.5 \mathrm{sec}$. The microsource dynamic responses including $d c$ voltage tracking, frequency synchronization, the power sharing as well as ac voltage

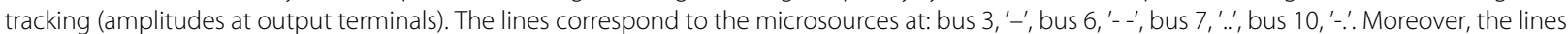

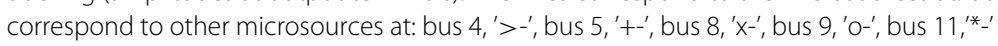



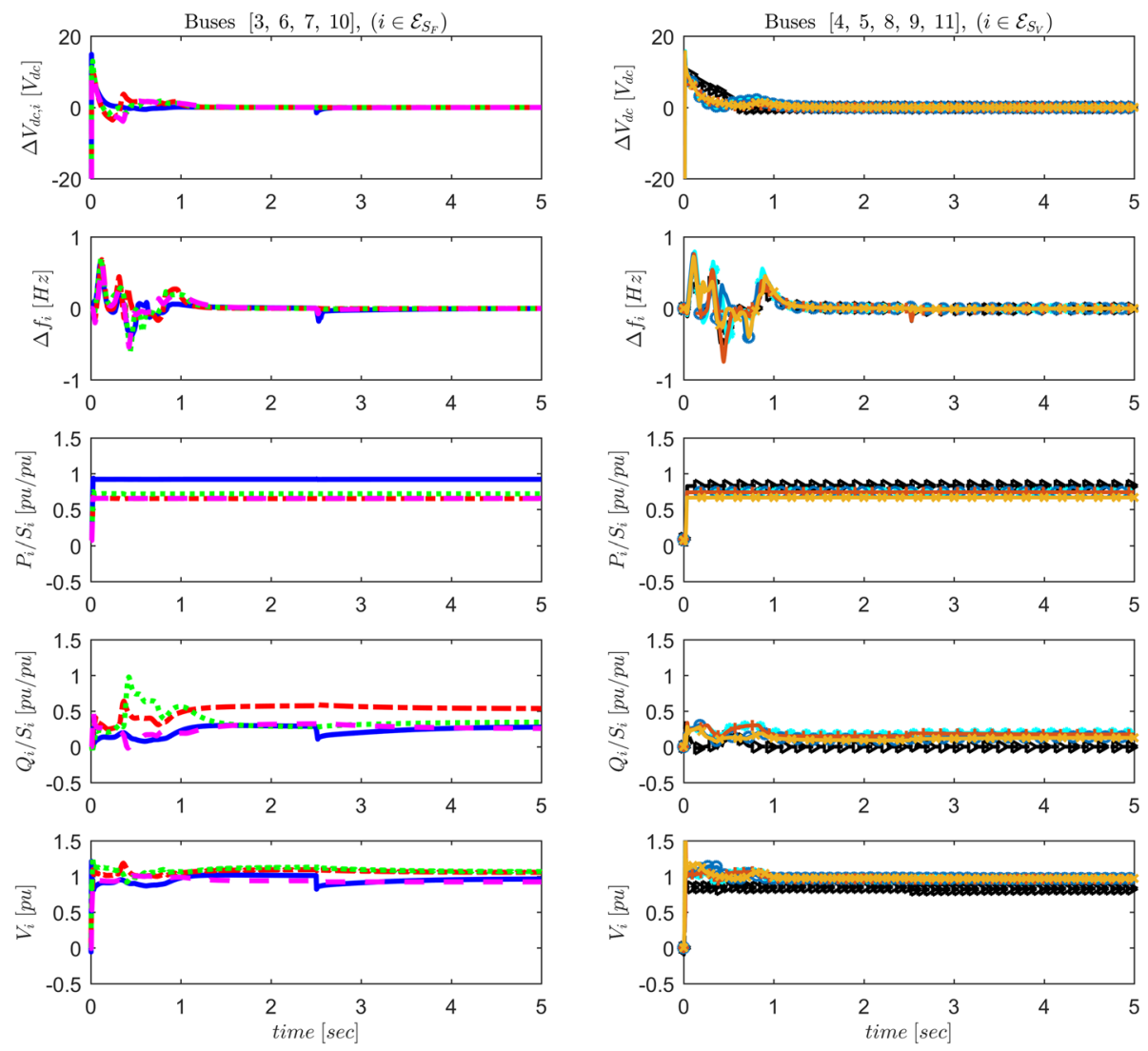

Fig. 14 Transition between islanded and grid-connected modes. The switching between slanded and grid-connected modes is occurred at time $2.5 \mathrm{sec}$. The microsource dynamic responses including $d c$ voltage tracking, frequency synchronization, the power sharing as well as ac voltage tracking (amplitudes at output terminals). The lines correspond to the microsources at: bus 3, '-', bus 6, '- - ', bus 7, '.., bus 10, '-.. Moreover, the lines

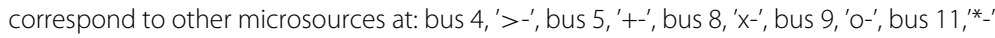

The initial conditions have been chosen arbitrarily. Hence, the simulation result shows the stability of the decentralized frequency and voltage control dynamics, as given in (77), (91) and (95). Furthermore, the simulation confirms that the decentralized control dynamics are suited to achieve the desired objective of frequency synchronization, voltage control and power sharing.

The simulation results demonstrate the effectiveness of the proposed control strategy. The designed controllers guarantee the power sharing and the frequency as well as voltage stability of the entire microgrid system.

\section{Conclusion}

The problem of decentralized control of inverter-based $a c$ micro-grid in different operation modes is addressed in this work. In addition, we focus on the problem of decentralized frequency and voltage control of the microgrid system without information exchange between the microsources. The design of passive dynamic controllers which ensure stability of the entire microgrid system is also addressed in this paper. Furthermore, to deal with nonlinear, interconnected and large-scale structure of micro-grid systems, the port-Hamiltonian formulation is used as a powerful tool to capture the dynamic of microgrid components and interconnection controllers. It is shown that both the microgrid dynamic as well as the controller designs admit a port-Hamiltonian representation which are then interconnected to obtain a closed-loop port-Hamiltonian system. Moreover, to deal with large signal stability problem of microgrid system, the shiftedHamiltonian energy function is served as a storage function to ensure incremental passivity of system. In addition, the contribution also expands the knowledge on the use of shifted-energy functions in the context of decentralized control of micro-grids. Moreover, it is shown that the aggregating of the microgrid dynamic and the decentralized controller dynamics satisfies the incremental passivity in the grid-connected and islanded modes. Finally, the effectiveness of the proposed controllers is evaluated through simulation studies. The different scenarios including grid-connected and islanded modes as well as transition between both modes are simulated. 
The simulation conforms that the decentralized control dynamics are suited to achieve the desired objective of frequency synchronization, voltage control and power sharing in the grid-connected and islanded modes. The simulation results demonstrate the effectiveness of the proposed control strategy.

Future works include attempting to add the hybrid microgrid systems and investigating large signal stability analysis as well as examining incremental passivity of entire system.

\section{Funding}

The authors declare that no fund is received from any financial or non-financial organization.

\section{Availability of data and materials}

Not applicable.

\section{Authors' contributions}

MFF contributed the concept of shifted-energy function and incremental passivity into the context of decentralized control of ac microgrid, modeled the microgrid components in port-Hamiltonian framework, designed decentralized passive dynamic controllers, analyzed the system stability and drafted the manuscript. AR and MG reviewed the manuscript. MFF and AR revised the manuscript. All authors read and approved the final manuscript.

\section{Authors' information}

M. Farokhian Firuzi is a doctoral candidate at the Department of Electrical and Electronics Engineering at Shiraz University of Technology, Iran. He received his B.Sc. in Electrical Engineering from the KNT University, Master of Electrical Engineering from Sharif University of Technology, Iran in 2005. He was a visiting student at Groningen University, The Netherlands. His main research interests are in decentralized Title Page Click here to access/download;Title Page;Cover letter.docx control and port-Hamiltonian modeling and analysis of complex networks with applications to micro-grids and power systems. His email address is m.farokhian@sutech.ac.ir. A. Roosta is a assistant professor at the Department of Electrical and Electronics Engineering at Shiraz University of Technology, Iran. He received his PhD degree in Electrical Engineering (Control) from the Institute of Polytechnic Grenoble, France in 2003. In addition, He received his Master in Electrical Engineering (Control) from Tehran University, Iran in 1991 and B.Sc. in Electrical Engineering from Chamran University, Iran in 1988. His main research interests are nonlinear control, fuzzy and neural control with applications in power systems engineering. His email address is roosta@sutech.ac.ir. M. Gitizadeh is a assistant professor at the Department of Electrical and Electronics Engineering at Shiraz University of Technology, Iran. He received his PhD degree and Master in Electrical Engineering (Power Systems) from Iran University of Science and Technology (IUST), Iran in 2009 and 2000 respectively. In addition, He received his B.Sc. in Electrical and Electronics Engineering from Shiraz University University, Iran in 1998. His main research interests are power system operation and control, reactive power control, distributed generation, smart-grids. His email address is gitizadeh@sutech.ac.ir.

\section{Competing interests}

The authors declare that they have no competing interests.

\section{Received: 5 June 2018 Accepted: 21 February 2019}

Published online: 30 March 2019

\section{References}

1. Hatziargyriou, N., Asano, H., Iravani, R., Marnay, C. (2007). Microgrids. IEEE Power and Energy Magazine, 5(4), 78-94.

2. Kroposki, B., Lasseter, R., Ise, T., Morozumi, S., Papatlianassiou, S., Hatziargyriou, N. (2008). Making microgrids work. IEEE power and energy magazine, 6(3), 40-53.

3. Guerrero, J.M., Chandorkar, M., Lee, T.L., Loh, P.C. (2013). Advanced control architectures for intelligent microgrids-part i: Decentralized and hierarchical control. IEEE Transactions on Industrial Electronics, 60(4), 12541262.
4. Chandorkar, M.C., Divan, D.M., Adapa, R. (1993). Control of parallel connected inverters in standalone ac supply systems. IEEE Transactions on Industry Applications, 29(1), 136-143.

5. Tuladhar, A., Jin, H., Unger, T., Mauch, K. (1997). Parallel operation of single phase inverter modules with no control interconnections, In Applied Power Electronics Conference and Exposition, 1997. APEC'97 Conference Proceedings 1997., Twelfth Annual, vol. 1 (pp. 94-100). Atlanta: IEEE.

6. Tuladhar, A., Jin, H., Unger, T., Mauch, K. (2000). Control of parallel inverters in distributed ac power systems with consideration of line impedance effect. IEEE Transactions on Industry Applications, 36(1), 131-138.

7. Majumder, R., Ghosh, A., Ledwich, G., Zare, F. (2009). Angle droop versus frequency droop in a voltage source converter based autonomous microgrid, In Power \& Energy Society General Meeting, 2009. PES'09. IEEE (pp. 1-8). Calgary: IEEE.

8. Majumder, R., Chaudhuri, B., Ghosh, A., Majumder, R., Ledwich, G., Zare, F. (2010). Improvement of stability and load sharing in an autonomous microgrid using supplementary droop control loop. IEEE Transactions on Power Systems, 25(2), 796-808.

9. Majumder, R., Ghosh, A., Ledwich, G., Zare, F. (2009). Load sharing and power quality enhanced operation of a distributed microgrid. IET Renewable Power Generation, 3(2), 109-119.

10. Li, Y.W., \& Kao, C.-N. (2009). An accurate power control strategy for powerelectronics-interfaced distributed generation units operating in a lowvoltage multibus microgrid. IEEE Transactions on Power Electronics, 24(12), 2977-2988

11. Guerrero, J.M., De Vicuna, L., Matas, J., Castilla, M., Miret, J. (2004). A wireless controller to enhance dynamic performance of parallel inverters in distributed generation systems. IEEE Transactions on Power Electronics, 19(5), 1205-1213.

12. Chiang, S.-J., Yen, C., Chang, K. (2001). A multimodule parallelable seriesconnected pwm voltage regulator. IEEE Transactions on Industrial Electronics, 48(3), 506-516.

13. Burup, U., Enjeti, P.N., Blaabjerg, F. (2001). A new space-vector-based control method for ups systems powering nonlinear and unbalanced loads. IEEE Transactions on Industry Applications, 37(6), 1864-1870.

14. Lee, T.-L., \& Cheng, P.-T. (2007). Design of a new cooperative harmonic filtering strategy for distributed generation interface converters in an islanding network. IEEE Transactions on Power Electronics, 22(5), 1919-1927.

15. Tanaka, K., Senjyu, T., Toma, S., Yona, A., Funabashi, T., Kim, C.-H. (2009) Decentralized voltage control in distribution systems by controlling reactive power of inverters, In Industrial Electronics, 2009. ISIE 2009. IEEE International Symposium On (pp. 1385-1390). Seoul: IEEE.

16. Riverso, S., Sarzo, F., Ferrari-trecate, G. (2014). Plug-and-play voltage and frequency control of islanded microgrids with meshed topology, (pp. 1-6): Citeseer.

17. Riverso, S., Sarzo, F., Ferrari-Trecate, G., et al (2015). Plug-and-play voltage and frequency control of islanded microgrids with meshed topology. IEEE Trans Smart Grid, 6(3), 1176-1184.

18. Riverso, S., Farina, M., Ferrari-Trecate, G. (2012). Plug-and-play decentralized model predictive control, In Decision and Control (CDC), 2012 IEEE 51st Annual Conference On (pp. 4193-4198). Maui: IEEE.

19. Riverso, S., Farina, M., Ferrari-Trecate, G. (2014). Plug-and-play model predictive control based on robust control invariant sets. Automatica, 50(8), 2179-2186.

20. Riverso, S. (2014). Distributed and plug-and-play control for constrained systems. doctoral dissertation. Universit'a degli studi di Pavia, Dipartimento di Informatica e Sistemistica.

21. Stoustrup, J. (2009). Plug \& play control: Control technology towards new challenges. European Journal of Control, 15(3-4), 311-330.

22. Vasquez, J.C., Guerrero, J.M., Savaghebi, M., Eloy-Garcia, J., Teodorescu, R. (2013). Modeling, analysis, and design of stationary-reference-frame droop-controlled parallel three-phase voltage source inverters. IEEE Transactions on Industrial Electronics, 60(4), 1271-1280.

23. Quesada, J., Sebastián, R., Castro, M., Sainz, J. (2014). Control of inverters in a low voltage microgrid with distributed battery energy storage. part $\mathrm{i}$ : Primary control. Electric Power Systems Research, 114, 126-135.

24. Golsorkhi, M.S., \& Lu, D.D. (2015). A control method for inverter-based islanded microgrids based on vi droop characteristics. IEEE Trans Power Deliv, 30(3), 1196-1204.

25. Zhu, Y., Zhuo, F., Wang, F., Liu, B., Gou, R., Zhao, Y. (2016). A virtual impedance optimization method for reactive power sharing in networked microgrid. IEEE Transactions on Power Electronics, 31(4), 2890-2904. 
26. Guan, Y., Guerrero, J.M., Zhao, X., Vasquez, J.C., Guo, X. (2016). A new way of controlling parallel-connected inverters by using synchronous-referenceframe virtual impedance loop_-part i: Control principle. IEEE Transactions on Power Electronics, 31(6), 4576-4593.

27. Taher, S.A., Zolfaghari, M., Cho, C., Abedi, M., Shahidehpour, M. (2017). A new approach for soft synchronization of microgrid using robust control theory. IEEE Transactions on Power Delivery, 32(3), 1370-1381.

28. Kim, J.H., Lee, Y.S., Kim, H.J., Han, B.M. (2017). A new reactive-power sharing scheme for two inverter-based distributed generations with unequal line impedances in islanded microgrids. Energies, 10(11), 1800.

29. Caracas, J.V., Farias, G.C., de Matos, J.G., Simões, F., Ribeiro, L.A. (2018). Adaptative droop control for balancing the state of charge of multiple energy storage systems in decentralized microgrids, In IECON 2018-44th Annual Conference of the IEEE Industrial Electronics Society (pp. 1658-1663). Washington, DC: IEEE.

30. Hou, X., Sun, Y., Han, H., Liu, Z., Yuan, W., Su, M. (2019). A fully decentralized control of grid-connected cascaded inverters. IEEE Transactions on Power Delivery, 10(1), 315-17. IEEE.

31. Sun, Y., Shi, G., Li, X., Yuan, W., Su, M., Han, H., Hou, X. (2018). An fp/q droop control in cascaded-type microgrid. IEEE Transactions on Power Systems, 33(1), 1136-1138.

32. Liu, J., Miura, Y., Ise, T. (2018). Cost-function-based microgrid decentralized control of unbalance and harmonics for simultaneous bus voltage compensation and current sharing. IEEE Transactions on Power Electronics, 99, $1-1$.

33. Haider, S., Li, G., Wang, K. (2018). A dual control strategy for power sharing improvement in islanded mode of ac microgrid. Protection and Control of Modern Power Systems, 3, 1-8.

34. Eskandari, M., Li, L., Moradi, M.H. (2018). Decentralized optimal servo control system for implementing instantaneous reactive power sharing in microgrids. IEEE Transactions on Sustainable Energy, 9(2), 525-537.

35. Sun, Q., Sun, Q., Qin, D. (2019). Adaptive fuzzy droop control for optimized power sharing in an islanded microgrid. Energies, 12(1), 45.

36. Kulkarni, O.V., Doolla, S., Fernandes, B. (2019). Simple controller configuration for decentralized parallel operation of inverters. IEEE Transactions on Power Electronics, 34(2), 1356-1369.

37. Chen, Z., Pei, X., Yang, M., Peng, L. (2019). An adaptive virtual resistor (avr) control strategy for low-voltage parallel inverters. IEEE Transactions on Power Electronics, 34(1), 863-876.

38. Yu, H., Tu, H., Lukic, S. (2018). A passivity-based decentralized control strategy for current-controlled inverters in ac microgrids, In 2018 IEEE Applied Power Electronics Conference and Exposition (APEC) (pp. 1399-1406). San Antonio: IEEE.

39. Li, L., Sun, Y., Liu, Z., Hou, X., Shi, G., Su, M. (2019). A decentralized control with unique equilibrium point for cascaded-type microgrid. IEEE Transactions on Sustainable Energy, 10(1), 324-326.

40. Chandorkar, M.C. (1995). Distributed Uninterruptible Power Supply Systems. Madison: University of Wisconsin-Madison.

41. Venkataramanan, G., \& Illindala, M. (2007). Small signal dynamics of inverter interfaced distributed generation in a chain-microgrid, In Power Engineering Society General Meeting, 2007. IEEE (pp. 1-6). Tampa: IEEE.

42. Mohamed, Y.A.-R.I., \& El-Saadany, E.F. (2008). Adaptive decentralized droop controller to preserve power sharing stability of paralleled inverters in distributed generation microgrids. IEEE Transactions on Power Electronics, 23(6), 2806-2816.

43. Iyer, S.V., Belur, M.N., Chandorkar, M.C. (2010). A generalized computational method to determine stability of a multi-inverter microgrid. IEEE Transactions on Power Electronics, 25(9), 2420-2432.

44. Tabesh, A., \& Iravani, R. (2009). Multivariable dynamic model and robust control of a voltage-source converter for power system applications. IEEE Transactions on Power Delivery, 24(1), 462-471.

45. Barklund, E., Pogaku, N., Prodanovic, M., Hernandez-Aramburo, C., Green, T.C. (2008). Energy management in autonomous microgrid using stabilityconstrained droop control of inverters. IEEE Transactions on Power Electronics, 23(5), 2346-52.

46. Delghavi, M.B., \& Yazdani, A. (2011). Islanded-mode control of electronically coupled distributed-resource units under unbalanced and nonlinear load conditions. IEEE Transactions on Power Delivery, 26(2), 661-673.

47. Mehrizi-Sani, A., \& Iravani, R. (2010). Potential-function based control of a microgrid in islanded and grid-connected modes. IEEE Transactions on Power Systems, 25(4), 1883-1891.
48. Soni, N., Doolla, S., Chandorkar, M.C. (2013). Improvement of transient response in microgrids using virtual inertia. IEEE Transactions on Power Delivery, 28(3), 1830-1838.

49. Jayawarna, N., Wu, X., Zhang, Y., Jenkins, N., Barnes, M. (2006). Stability of a microgrid. IET Conference Proceedings, (pp. 316-20(4)). Dublin: IET.

50. Katiraei, F., Iravani, M., Lehn, P. (2007). Small-signal dynamic model of a micro-grid including conventional and electronically interfaced distributed resources. IET generation, transmission \& distribution, 1(3), 369378.

51. Pogaku, N., Prodanovic, M., Green, T.C. (2007). Modeling, analysis and testing of autonomous operation of an inverter-based microgrid. IEEE Transactions on Power Electronics, 22(2), 613-625.

52. Bottrell, N., Prodanovic, M., Green, T.C. (2013). Dynamic stability of a microgrid with an active load. IEEE Transactions on Power Electronics, 28(11), 5107-5119.

53. Nasr-Azadani, E., Cañizares, C.A., Olivares, D.E., Bhattacharya, K. (2014). Stability analysis of unbalanced distribution systems with synchronous machine and dfig based distributed generators. IEEE Transactions on Smart Grid, 5(5), 2326-2338.

54. Sun, J. (2009). Small-signal methods for ac distributed power systems-a review. IEEE Transactions on Power Electronics, 24(11), 2545-2554.

55. Sun, J. (2011). Impedance-based stability criterion for grid-connected inverters. IEEE Transactions on Power Electronics, 26(11), 3075.

56. Rasheduzzaman, M. (2015). Small signal modeling and analysis of microgrid systems. Missouri University of Science and Technology.

57. Alaboudy, A.K., Zeineldin, H.H., Kirtley, J. (2012). Microgrid stability characterization subsequent to fault-triggered islanding incidents. IEEE Transactions on Power Delivery, 27(2), 658-669.

58. Kamel, R.M., Chaouachi, A., Nagasaka, K. (2011). Detailed analysis of microgrid stability during islanding mode under different load conditions. Engineering, 3(05), 508.

59. Azadani, E.N., Canizares, C., Bhattacharya, K. (2012). Modeling and stability analysis of distributed generation, In Power and Energy Society General Meeting, 2012 IEEE (pp. 1-8). San Diego: IEEE.

60. Farrokhabadi, M., Cañizares, C.A., Bhattacharya, K. (2017). Frequency control in isolated/islanded microgrids through voltage regulation. IEEE Transactions on Smart Grid, 8(3), 1185-1194.

61. Vasquez, J.C., Guerrero, J.M., Luna, A., Rodríguez, P., Teodorescu, R. (2009) Adaptive droop control applied to voltage-source inverters operating in grid-connected and islanded modes. IEEE Transactions on Industrial Electronics, 56(10), 4088-4096.

62. Diaz, G., Gonzalez-Moran, C., Gomez-Aleixandre, J., Diez, A. (2010). Scheduling of droop coefficients for frequency and voltage regulation in isolated microgrids. IEEE Transactions on Power Systems, 25(1), 489-496.

63. Kim, J., Guerrero, J.M., Rodriguez, P., Teodorescu, R., Nam, K. (2011). Mode adaptive droop control with virtual output impedances for an inverterbased flexible ac microgrid. IEEE Transactions on power electronics, 26(3), 689-701.

64. Wang, Z., Xia, M., Lemmon, M. (2013). Voltage stability of weak power distribution networks with inverter connected sources, In American Control Conference (ACC), 2013 (pp. 6577-6582): IEEE.

65. Schiffer, J., Ortega, R., Astolfi, A., Raisch, J., Sezi, T. (2014). Conditions for stability of droop-controlled inverter-based microgrids. Automatica, 50(10), 2457-2469.

66. Sun, Y., Huang, W., Wang, G., Wang, W., Wang, L., Zhang, D. (2012). Study of control strategy of dg based on nonlinear droop characteristic, In Electricity Distribution (CICED), 2012 China International Conference On (pp. 1-4). Shanghai: IEEE.

67. Zhang, Y. (2017). Capacity-based adaptive droop control for battery energy storage operation, In Power \& Energy Society General Meeting, 2017 IEEE (pp. 1-5). Chicago: IEEE.

68. Zhang, T., Orr, J.A., Emanuel, A.E. (2018). Adaptable energy storage system control for microgrid stability enhancement, In 2018 IEEE Power \& Energy Society General Meeting (PESGM) (pp. 1-5). Portland: IEEE.

69. Majumder, R. (2013). Some aspects of stability in microgrids. IEEE Transac tions on Power Systems, 28(3), 3243-3252

70. Shuai, Z., Sun, Y., Shen, Z.J., Tian, W., Tu, C., Li, Y., Yin, X. (2016). Microgrid stability: Classification and a review. Renewable and Sustainable Energy Reviews, 58, 167-179.

71. Solano, J., Rey, J.M., Bastidas-Rodríguez, J.D., Hernández, A.I. (2019). Stability issues in microgrids, In Microgrids Design and Implementation (pp. 287 310). Cham: Springer. 
72. Pogaku, N., Prodanovic, M., Green, T.C. (2007). Modeling, analysis and testing of autonomous operation of an inverter-based microgrid. IEEE Transactions on Power Electronics, 22(2), 613-625.

73. Karimi, H., Davison, E.J., Iravani, R. (2010). Multivariable servomechanism controller for autonomous operation of a distributed generation unit: Design and performance evaluation. IEEE Transactions on Power Systems, 25(2), 853-865.

74. Karimi, H., Nikkhajoei, H., Iravani, R. (2008). Control of an electronicallycoupled distributed resource unit subsequent to an islanding event. IEEE Transactions on Power Delivery, 23(1), 493-501.

75. Karimi, H., Yazdani, A., Iravani, R. (2011). Robust control of an autonomous four-wire electronically-coupled distributed generation unit. IEEE Transactions on Power Delivery, 26(1), 455-466.

76. Karimi, H., Yazdani, A., Iravani, R. (2011). Robust control of an autonomous four-wire electronically-coupled distributed generation unit. IEEE Transactions on Power Delivery, 26(1), 455-466.

77. Sadabadi, M.S., Karimi, A., Karimi, H. (2015). Fixed-order decentralized/distributed control of islanded inverter-interfaced microgrids. Control Engineering Practice, 45, 174-193.

78. Babazadeh, M., \& Karimi, H. (2013). A robust two-degree-of-freedom control strategy for an islanded microgrid. IEEE transactions on power delivery, 28(3), 1339-1347.

79. Bahrani, B., Saeedifard, M., Karimi, A., Rufer, A. (2013). A multivariable design methodology for voltage control of a single-dg-unit microgrid. IEEE Transactions on Industrial Informatics, 9(2), 589-599.

80. Serra, F.M., \& De Angelo, C.H. (2017). Ida-pbc controller design for grid connected front end converters under non-ideal grid conditions. Electric Power Systems Research, 142, 12-19.

81. Weiss, G., Zhong, Q.-C., Green, T.C., Liang, J. (2004). $H_{\infty}$ repetitive control of dc-ac converters in microgrids. IEEE Transactions on Power Electronics, 19(1), 219-230.

82. Li, Y.W., Vilathgamuwa, D.M., Loh, P.C. (2007). Robust control scheme for a microgrid with pfc capacitor connected. IEEE Transactions on Industry Applications, 43(5), 1172-1182.

83. Moradi, R., Karimi, H., Karimi-Ghartemani, M. (2010). Robust decentralized control for islanded operation of two radially connected dg systems, In Industrial Electronics (ISIE), 2010 IEEE International Symposium On (pp. 22722277). Bari: IEEE.

84. Schiffer, J., Anta, A., Trung, T.D., Raisch, J., Sezi, T. (2012). On power sharing and stability in autonomous inverter-based microgrids, In Decision and Control (CDC), 2012 IEEE 51st Annual Conference On (pp. 1105-1110). Maui: IEEE.

85. Andreasson, M., Sandberg, H., Dimarogonas, D.V., Johansson, K.H. (2012). Distributed integral action: Stability analysis and frequency control of power systems, In Decision and Control (CDC), 2012 IEEE 51st Annual Conference On (pp. 2077-2083). Maui: IEEE.

86. Taher, S.A., \& Zolfaghari, M. (2014). Designing robust controller to improve current-sharing for parallel-connected inverter-based dgs considering line impedance impact in microgrid networks. International Journal of Electrical Power \& Energy Systems, 63, 625-644.

87. van der Schaft, A., Jeltsema, D., et al (2014). Port-hamiltonian systems theory: An introductory overview. Foundations and Trends ${ }^{\circledR}$ in Systems and Control, 1(2-3), 173-378.

88. Van der Schaft, A. (2017). L2-gain and Passivity Techniques in Nonlinear Control, vol. 218. Cham: Springer.

89. Duindam, V., Macchelli, A., Stramigioli, S., Bruyninckx, H. (2009). Modeling and Control of Complex Physical Systems: the port-Hamiltonian Approach. Berlin: Springer.

90. Zonetti, D., Ortega, R., Benchaib, A. (2015). Modeling and control of hvdc transmission systems from theory to practice and back. Control Engineering Practice, 45, 133-146.

91. Zonetti, D. (2016). Energy-based modelling and control of electric power systems with guaranteed stability properties. PhD thesis, Université ParisSaclay. Universit'e Paris-Saclay.

92. Fiaz, S., Zonetti, D., Ortega, R., Scherpen, J.M., van Der Schaft, A. (2013). A port-hamiltonian approach to power network modeling and analysis. European Journal of Control, 19(6), 477-485.

93. van der Schaft, A., \& Stegink, T. (2016). Perspectives in modeling for control of power networks. Annual Reviews in Control, 41, 119-132.
94. De Persis, C., Monshizadeh, N., Schiffer, J., Dörfler, F. (2016). A lyapunov approach to control of microgrids with a network-preserved differentialalgebraic model, In Proceedings of IEEE Conference on Decision and Control (pp. 2595-2600). Las Vegas: IEEE.

95. Yan-Hong, L., Chun-Wen, L., Yu-Zhen, W. (2009). Decentralized excitation control of multi-machine multi-load power systems using hamiltonian function method. Acta Automatica Sinica, 35(7), 919-925.

96. Xi, Z., Cheng, D., Lu, Q., Mei, S. (2002). Nonlinear decentralized controller design for multimachine power systems using hamiltonian function method. Automatica, 38(3), 527-534.

97. Arghir, C., Groß, D., Dörfler, F. (2016). On the steady-state behavior of a nonlinear power network model. IFAC-PapersOnLine, 49(22), 61-66.

98. Jayawardhana, B., Ortega, R., Garcia-Canseco, E., Castanos, F. (2007). Passivity of nonlinear incremental systems: Application to pi stabilization of nonlinear rlc circuits. Systems \& Control Letters, 56(9-10), 618-622.

99. Desoer, C.A., \& Vidyasagar, M. (2009). Feedback Systems: Input-output Properties. Philadelphia: SIAM.

100. Lasseter, R.H. (2002). Microgrids, In Power Engineering Society Winter Meeting, 2002. IEEE, vol.1 (pp. 305-308). New York: IEEE.

101. Shahidehpour, M. (2010). Role of smart microgrid in a perfect power system, In Power and Energy Society General Meeting, 2010 IEEE (pp. 1-1). Providence: IEEE.

102. Farhangi, H. (2010). The path of the smart grid. IEEE Power and Energy Magazine, 8(1), 18-28.

103. Khodayar, M.E., Barati, M., Shahidehpour, M. (2012). Integration of high reliability distribution system in microgrid operation. IEEE Transactions on Smart Grid, 3(4), 1997-2006.

104. Jouini, T., Arghir, C., Dörfler, F. (2016). Grid-friendly matching of synchronous machines by tapping into the dc storage. IFAC-PapersOnLine, 49(22), 192-197.

105. Rudion, K., Orths, A., Styczynski, Z.A., Strunz, K. (2006). Design of benchmark of medium voltage distribution network for investigation of $\mathrm{dg}$ integration, In Power Engineering Society General Meeting, 2006. IEEE (p. 6). Montreal: IEEE.

\section{Submit your manuscript to a SpringerOpen ${ }^{\circ}$ journal and benefit from:}

- Convenient online submission

- Rigorous peer review

- Open access: articles freely available online

- High visibility within the field

- Retaining the copyright to your article

Submit your next manuscript at $>$ springeropen.com 